\title{
Multiple sweeping using the Denavit-Hartenberg representation method
}

\author{
K. Abdel-Malek ${ }^{\mathrm{a}, *}$, S. Othman ${ }^{\mathrm{b}}$ \\ ${ }^{\mathrm{a}}$ Department of Mechanical Engineering and Center for Computer-Aided Design, The University of Iowa, Iowa City, IA 52242, USA \\ ${ }^{\mathrm{b}}$ US National Advanced Driving Simulator and Simulation Center, 2401 Oakdale Avenue, The University of Iowa, Iowa City, IA 52242, USA
}

Received 14 February 1999; received in revised form 15 June 1999; accepted 23 June 1999

\begin{abstract}
The method of consecutive revolving or extrusion of a geometric entity in a CAD system is typically used by a designer to represent complex solids. While it is evident that consecutive sweeping is a very effective tool, it has been restricted by the associated difficult mathematics in representing consecutive sweeps and in analyzing the resulting equations. The Denavit-Hartenberg (DH) method, first introduced in 1955 in the field of kinematics to mathematically relate motion between two coordinate frames, provides an effective formulation for characterizing the resulting solid model. Design variations imposed on sweep properties are easily propagated through the formulation to update the solid. This paper illustrates the use of the DH formulation for multiple sweeps and demonstrates the method through examples. (C) 1999 Elsevier Science Ltd. All rights reserved.
\end{abstract}

Keywords: Solid modeling; Denavit-Hartenberg; Sweeping; Computer-aided design

\section{Introduction}

Solid modeling provides the most complete representation of an object whereby quantities such as mass, center of gravity, and moments of inertia can readily be computed. A number of different schemes have been used to represent solids such as primitive instancing, cell decomposition, sweep representations, constructive solid geometry (CSG), and boundary representations (B-rep). Most of the times, a combination of the above methods is used to generate complex solids. The concept of sweeps as a tool to generate solids is commonly used by designers but only for a minimum number of sweep variables. Solid modeling is computationally expensive and efficient methods of model construction are needed to reduce computational time.

A detailed review of major developments of solid modeling methods is presented by Hoffman [1]. Hoffman states:

The mathematics of sweeping is more delicate and demanding than it might seem at first glance. ...Usually, there is no closed-form mathematical description of the surface bounding the swept volume.

However, recent work [2], presents a closed-form mathematical method for the determination of surfaces bounding

\footnotetext{
* Corresponding author. Tel.: + 1-319-335-5676; fax: + 1-319-3355669 .

E-mail addresses: amalek@engineering.uiowa.edu (K. Abdel-Malek), othman@nads-sc.uiowa.edu (S. Othman)
}

the volume. The work presented in this paper is formalism for the development of a systematic method for computing the swept volume generated by consecutive sweeping.

While there has been an extensive amount of work pertaining to analysis and formulations for swept volume techniques, we cite those that have appeared in recent years and refer the reader to visit the web page dedicated to swept volumes research and applications [www.icaen.uiowa.ed/am/sweep/ sweept]. Recent work in the field include Refs. [3-16].

The consecutive sweep of a geometric entity in a Computer-Aided Design (CAD) environment is a very effective method for producing complex solids as was shown in recent work by [17]. In this paper, we present an effective method to formulate the sweep equation for multiple revolve and extrude operations that would serve as a preprocessor module in a CAD environment as illustrated in Fig. 1.

A circle, for example, is extruded along an axis to produce a cylindrical surface characterized by two parameters. This surface is revolved about another axis to yield a volume characterized by three parameters. Again, the volume is now extruded to yield a more complex solid in four parameters. This design method provides a level of flexibility that is not evidenced by any of the aforementioned modeling methods but that can be used as a complementary aid to these methods. While this multiple sweeping idea is often more difficult to visualize, it provides a new technique for design, especially when the outcome is still uncertain and further work on the solid may be required. The proposed method will formulate this multiple sweeping 


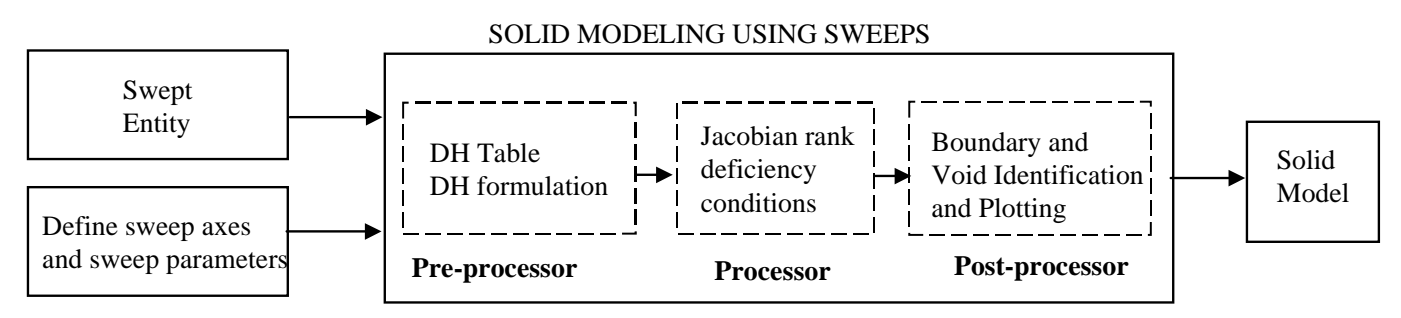

Fig. 1. DH formulation as a module for solid modeling.

using a systematic mathematical approach that would maintain the sweep variables in the final expression. As a result, a change in the design after the initial sweeps have been selected is now possible and can easily be performed in analogy with existing parametric methods.

It should also be noted here that sweeping, as in the field of kinematics, is not limited to revolve and extrude operations (revolute and prismatic joints). Any sweep analogous to a joint in kinematics can be modeled. For example, a spherical joint is a combination of three intersecting revolute joints, and a cylindrical joint is a combination of a revolute and a prismatic joint. Similarly, other types of sweeping (such as the general screw motion) can be represented as well.

The concept of multiple sweeping to generate solid models has not yet been addressed in the literature because of the relatively complex mathematics. Envelope theory [18], for example, typically used in the analysis of swept volumes, is limited to three parameters. Similarly, the elegant method of sweep differential equation [19-21], although has treated several important issues pertaining to computer aided geometric design using swept volumes, has not yet been applied to consecutive sweeps of an object. The Lie group of Euclidean motions is 6-D (6-parameters) and the swept volume of a 3-D object can usually be expressed in terms of 4 parameters. Therefore, the potential for SEDE method to address consecutive sweeping exists.

The Denavit-Hartenberg (DH) method [22-24] was created to systematically establish a coordinate system in each link of an articulated kinematic chain. A mechanism, composed of several links can be represented using the DH method to relate the position and orientation of the last link to the first. Because of the DH method, displacement and angle parameters of the joints connecting two links are considered as variables in the formulation. In this work, the $\mathrm{DH}$ formulation will be used to characterize the variables representing each sweep. Indeed, the DH formulation will:

1. provide a systematic method for locating coordinate systems for each sweep operation;

2. characterize each sweep using four independent parameters;

3. formulate the solid model in terms of these variables such that varying any or all of the parameters can automatically change the solid model;

4. provide an ability for computing solid mass properties (volume and moments of inertia).

\section{The Denavit-Hartenberg representation method}

In studying the kinematic motion between two jointed links, the DH-method defines the position and orientation of two consecutive links in a chain, link $i$ with respect to link $(i-1)$, using a $(4 \times 4)$ homogeneous transformation matrix. For our purposes, an extrude, revolve, or any sweep operation, will be represented by a specific type of kinematic pair (a joint). The extrusion of a geometric entity along an axis is identical to rigid body motion of an entity along a prismatic joint, while revolving a geometric entity about an axis is identical to rigid body motion about a revolute joint. The consecutive sweep operations provide a powerful method for solid modeling.

For example, consider the extrusion of a curve $\mathbf{c}(t)$ shown in Fig. 2(a), along axis $\mathbf{a}_{1}$ to generate a surface $\mathbf{s}(t, u)$, parameterized in two variables. This surface is then revolved along a curve (about axis $\mathbf{a}_{2}$ ) to generate a volume $\mathbf{v}(t, u, v)$ parameterized in three variables and shown in Fig. 2(b).

The volume is then swept again along a curve (about axis

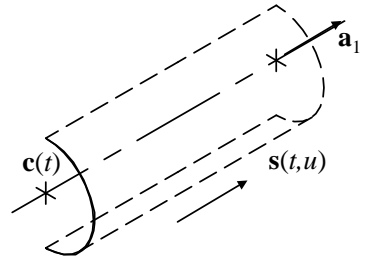

(a)

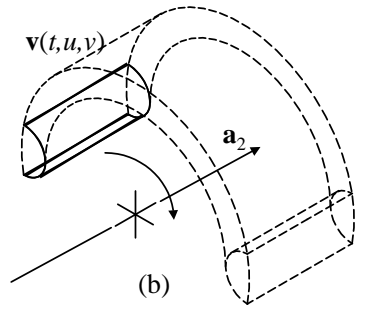

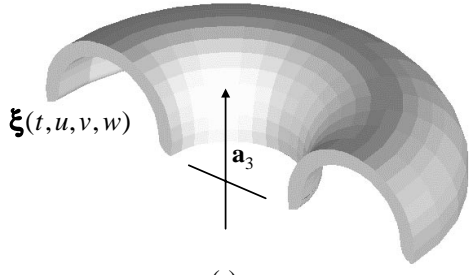

(c)

Fig. 2. (a) Sweeping a curve along an axis. (b) Sweeping a surface along a curve. (c) A sweep of the resulting volume along a curve. 


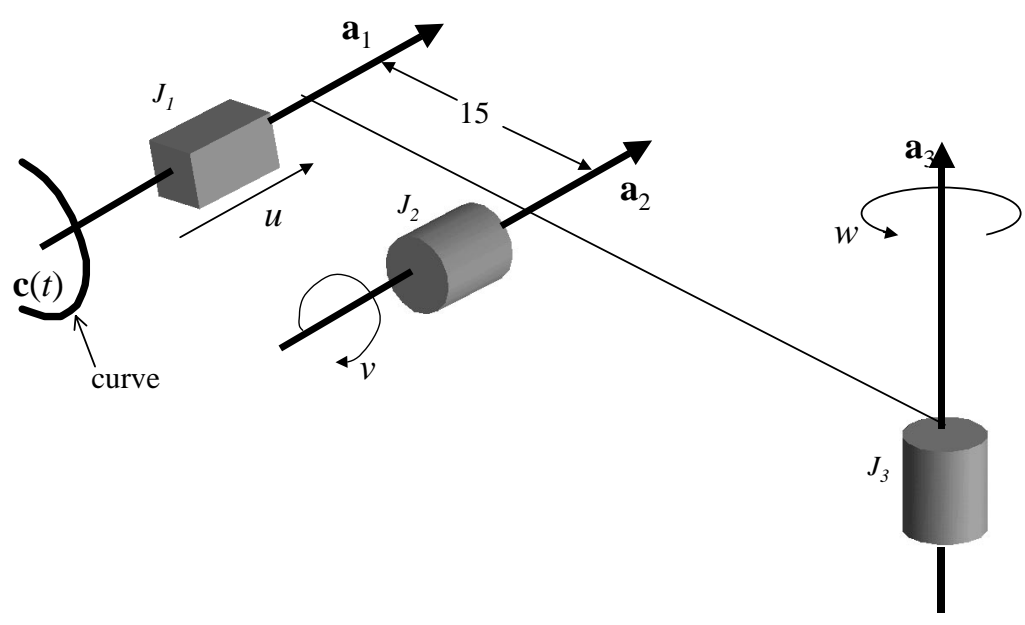

Fig. 3. Modeling of the sweep.

$\left.\mathbf{a}_{3}\right)$ to generate a more complex shape parameterized in four variables $\boldsymbol{\xi}(t, u, v, w)$ shown in Fig. 2(c).

Consecutive sweep operations are represented by a number of joints. Sweeping along axis $\mathbf{a}_{1}$ is represented by a translating joint $J_{1}$ shown in Fig. 3. Revolving about $\mathbf{a}_{2}$ is represented by a revolute joint $J_{2}$ and revolving about $\mathbf{a}_{3}$ is represented by $J_{3}$.

For a pair of consecutive sweeps about any two axes, the $\mathrm{DH}$ representation provides a systematic method for the establishing of a homogeneous transformation matrix between them. The result is a single equation of the solid as a function of the sweep parameters. A change in one of the parameters is propagated into the solid without the need for recreating the sweep operations. This important notion will become more evident in the following sections.

\subsection{Minimal representation between two coordinate frames}

In this section, we will use the property that two coordinate systems satisfying the property that the $\mathbf{x}$-axis of each of the systems is orthogonal to the $\mathbf{z}$-axis of the other system are related by a Euclidean motion depending on four parameters, rather than six parameters necessary for a pair in general position. Consider two coordinate frames in space, the first denoted by $\mathbf{x}_{0}-\mathbf{y}_{0}-\mathbf{z}_{0}$ with origin at $O_{0}$ and the second by $\mathbf{x}_{1}-\mathbf{y}_{1}-\mathbf{z}_{1}$ with origin at $O_{1}$. Let $P$ be an arbitrary point in space. Let ${ }^{0} \mathbf{v}$ be the vector coordinates of $P$ with respect to the $\mathbf{x}_{0}-\mathbf{y}_{0}-\mathbf{z}_{0}$ reference frame. Let ${ }^{1} v$ be the vector of coordinates of $P$ with respect to frame 1 . Let ${ }^{0} b_{1}$ be the vector describing the origin of frame 1 with respect to frame 0 as shown in Fig. 4.

Using geometry, the position of point $P$ with respect to frame 0 can be expressed as

${ }^{0} \mathbf{v}={ }^{0} \mathbf{b}_{1}+{ }^{0} \mathbf{R}_{1}{ }^{1} \mathbf{v}$

where ${ }^{0} \mathbf{R}_{1}=\left[\begin{array}{lll}\mathbf{x}_{1} & \mathbf{y}_{1} & \mathbf{z}_{1}\end{array}\right]$ is the rotation matrix relating the orientation of both frames. In order to achieve a compact representation and to simplify the mathematics between subsequent coordinate frames, the homogeneous representation of a generic vector $\mathbf{v}$ can be introduced as the vector $\tilde{\mathbf{v}}=\left[\mathbf{v}^{\mathrm{T}} 1\right]^{\mathrm{T}}$ formed by adding a fourth unit component. By adopting this representation for the vectors ${ }^{0} \mathbf{v}$ and ${ }^{1} \mathbf{v}$, the coordinate transformation can be written

${ }^{0} \mathbf{T}_{1}=\left[\begin{array}{cc}{ }^{0} \mathbf{R}_{1} & { }^{0} \mathbf{b}_{1} \\ \mathbf{0} & 1\end{array}\right]$

and is termed the homogeneous transformation matrix. Hence, transforming a vector from one coordinate frame to another is written as

${ }^{0} \tilde{\mathbf{v}}={ }^{0} \mathbf{T}_{1}{ }^{1} \tilde{\mathbf{v}}$

In order to generate the matrix relating any two sweeps, a minimal representation of only four parameters is necessary to describe one coordinate system with respect to the other. These four parameters determine the position and orientation of a coordinate axis with respect to another using a $(4 \times$ 4) homogeneous transformation matrix. To establish this matrix, it is possible to observe that a vector resolved in the $i$ th coordinate system ${ }^{i} \mathbf{v}$ may be expressed in the $(i-$ 1)th coordinate system ${ }^{i-1} \mathbf{v}$ by performing four successive transformations as follows:

1. A rotation about the $\mathbf{z}_{i-1}$ axis by an angle of $\theta_{i}$ to align

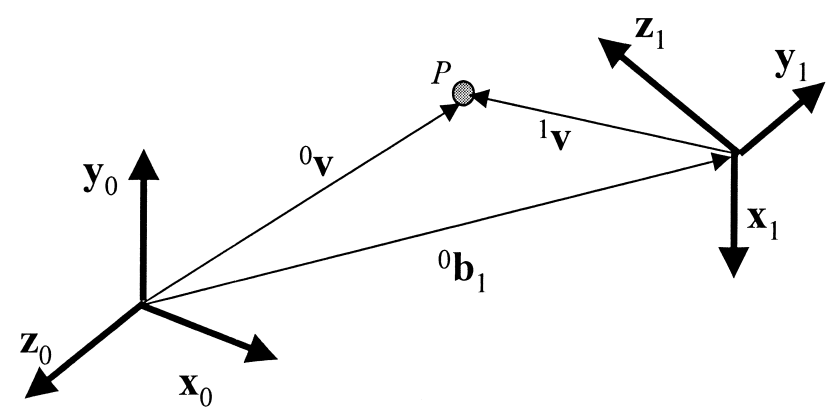

Fig. 4. Representation of a point $P$. 


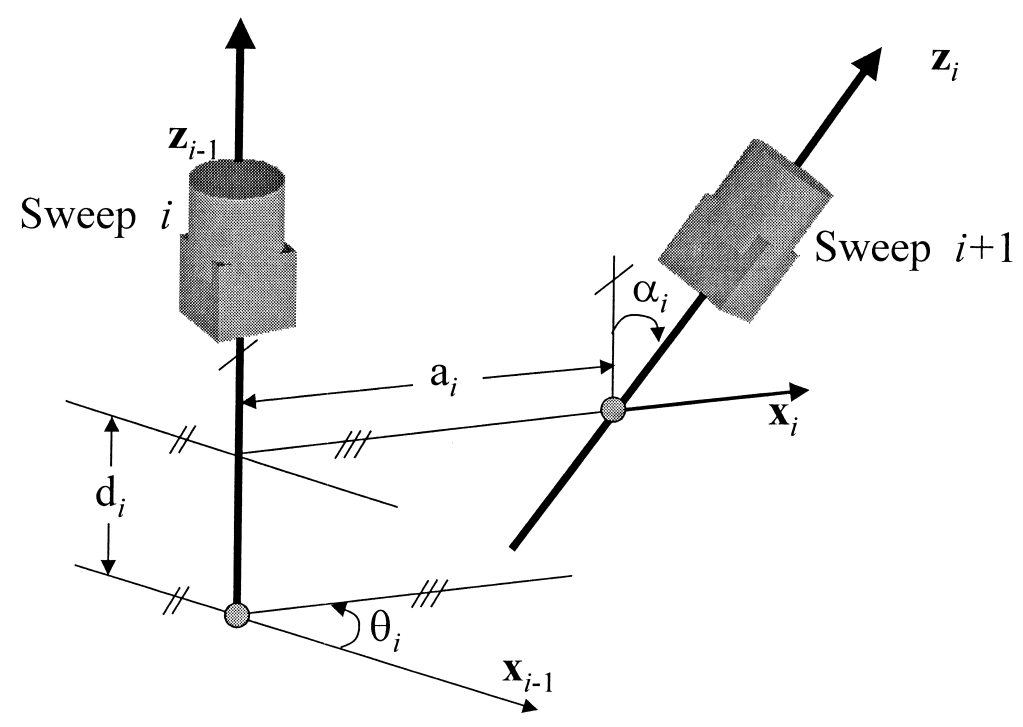

Fig. 5. Establishing coordinate systems and the four D-H parameters.

the $\mathbf{x}_{i-1}$ axis with the $\mathbf{x}_{i}$ axis (as shown in Fig. $5, \mathbf{x}_{i-1} \| \mathbf{x}_{i}$ and pointing in the same direction). Note that the joint representing each sweep in this case need not be prismatic or revolute and hence the revolute-prismatic symbol in Fig. 5.

2. A translation matrix along the $\mathbf{z}_{i-1}$ by a distance of $d_{i}$ units to make $\mathbf{x}_{i-1}$ and $\mathbf{x}_{i}$ aligned.

3. A translation matrix along the $\mathbf{x}_{i}$ axis by a distance of $a_{i}$ units to make the two origins of the $i$ and $(i-1)$ systems coincide (the $\mathbf{x}_{i}$ and the $\mathbf{x}_{i-1}$ will also be aligned).
Each step above is expressed by a basic homogeneous rotation or translation matrix (e.g. $\mathbf{T}_{z, d}$ denotes a translation matrix along $\mathbf{z}$ with $d$ units). The product of these four matrices results in a composite homogeneous transformation matrix relating the $(i-1)$ frame to the $i$ th frame as

${ }^{i-1} \mathbf{T}_{i}=\mathbf{T}_{z, d} \mathbf{T}_{z, \theta} \mathbf{T}_{x, a} \mathbf{T}_{x, \alpha}$

Substituting for each basic transformation matrix, Eq. (1) can be written as

${ }^{(i-1)} \mathbf{T}_{i}=\left[\begin{array}{cccc}1 & 0 & 0 & 0 \\ 0 & 1 & 0 & 0 \\ 0 & 0 & 1 & d_{i} \\ 0 & 0 & 0 & 1\end{array}\right]\left[\begin{array}{cccc}\cos \theta_{i} & -\sin \theta_{i} & 0 & 0 \\ \sin \theta_{i} & \cos \theta_{i} & 0 & 0 \\ 0 & 0 & 1 & 0 \\ 0 & 0 & 0 & 1\end{array}\right]\left[\begin{array}{cccc}1 & 0 & 0 & a_{i} \\ 0 & 1 & 0 & 0 \\ 0 & 0 & 1 & 0 \\ 0 & 0 & 0 & 1\end{array}\right]\left[\begin{array}{cccc}1 & 0 & 0 & 0 \\ 0 & \cos \alpha_{i} & -\sin \alpha_{i} & 0 \\ 0 & \sin \alpha_{i} & \cos \alpha_{i} & 0 \\ 0 & 0 & 0 & 1\end{array}\right]$

$$
=\left[\begin{array}{cccc}
\cos \theta_{i} & -\cos \alpha_{i} \sin \theta_{i} & \sin \alpha_{i} \sin \theta_{i} & a_{i} \cos \theta_{i} \\
\sin \theta_{i} & \cos \alpha_{i} \cos \theta_{i} & -\sin \alpha_{i} \cos \theta_{i} & a_{i} \sin \theta_{i} \\
0 & \sin \alpha_{i} & \cos \alpha_{i} & d_{i} \\
0 & 0 & 0 & 1
\end{array}\right]
$$

4. A rotation matrix about the $\mathbf{x}_{i}$ axis by an angle $\alpha_{i}$ to coincide the two coordinate systems.

Therefore, the parameters $\theta_{i}, d_{i}, a_{i}, \alpha_{i}$ characterize the position and orientation of any two consecutive sweeps. This is possible only because of the unique formulation whereby the $z$-axes are located along the sweep operation (joint axis) and the $x$-axes are perpendicular to any two consecutive sweeps. The rules for affixing these axes are addressed in the following section.
Define a set of generalized coordinates $q_{1}, q_{2}, \ldots, q_{n}$ that represent the variables in ${ }^{i-1} \mathbf{T}_{i}$ and those of the swept entity, where $q_{i}=\theta_{i}$ for a revolve action and $q_{i}=d_{i}$ for an extrude action. Note that these variables have inequality constraints imposed on them in the form of

$q_{i}^{\min } \leq q_{i} \leq q_{i}^{\max }$

For any sequence of consecutive sweeps, two reference frames are represented by the multiplication of their 


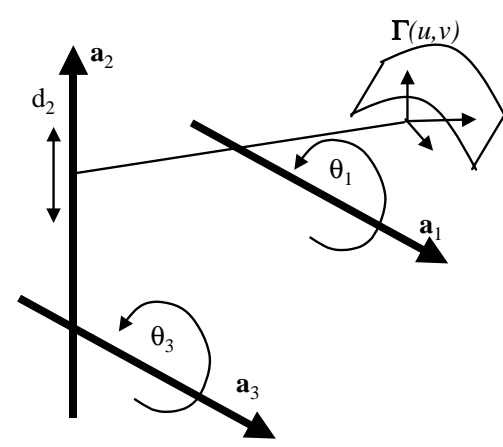

(a)

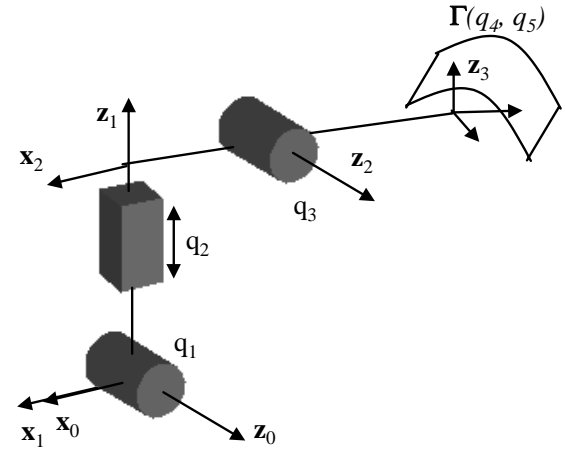

(b)

Fig. 6. (a) A surface subjected to a revolve, an extrude and a second revolve sweeping. (b) Replacing sweeps by joints and using the rules for affixing frames.

respective transformation. To obtain the transformation matrix relating the global frame (frame 0 ) to the $n$th frame, multiply the individual transformations as

${ }^{0} \mathbf{T}_{n}\left(q_{1}, \ldots, q_{n}\right)={ }^{0} \mathbf{T}_{1}\left(q_{1}\right)^{1} \mathbf{T}_{2}\left(q_{2}\right) \ldots{ }^{n-1} \mathbf{T}_{n}\left(q_{n}\right)=\prod_{j=1}^{n}{ }^{j-1} \mathbf{T}_{j}\left(q_{j}\right)$

where

${ }^{0} \mathbf{T}_{n}\left(q_{1}, \ldots, q_{n}\right)=\left[\begin{array}{cc}{ }^{0} \mathbf{R}_{n} & { }^{0} \mathbf{p}_{n} \\ \mathbf{0} & 1\end{array}\right]$,

and ${ }^{0} \mathbf{R}_{n}$ is a $(3 \times 3)$ rotation matrix, ${ }^{0} \mathbf{p}_{n}$ is a position vector from the origin of frame 0 to the origin of the $n$th frame. For $n$-sweep actions, the resulting transformation matrix with respect to frame 0 is ${ }^{0} \mathbf{T}_{n}\left(q_{1}, \ldots, q_{n}\right)$.For a sweep of the geometric entity $\boldsymbol{\Gamma}\left(q_{n+1}, \ldots, q_{n+m}\right)$, where $m$ is the number of parameters characterizing the swept entity (example of $\Gamma$ is a cylindrical surface given by $\left.\boldsymbol{\Gamma}(u, v)=[5 \cos u 5 \sin u v]^{\mathrm{T}}\right)$. The totality of points in the swept volume representing every point in the solid model is $\xi(\mathbf{q})$ computed from

$$
\begin{aligned}
{\left[\boldsymbol{\xi}^{\mathrm{T}}(\mathbf{q}) 1\right]^{\mathrm{T}} } & ={ }^{0} \mathbf{T}_{n}\left(q_{1}, \ldots, q_{n}\right)\left[\begin{array}{c}
\boldsymbol{\Gamma}\left(q_{n+1}, \ldots, q_{n+m}\right) \\
1
\end{array}\right] \\
& =[x(\mathbf{q}) y(\mathbf{q}) z(\mathbf{q}) \mathbf{1}]^{\mathrm{T}}
\end{aligned}
$$

where $\boldsymbol{\xi}(\mathbf{q})=\left[\begin{array}{ll}x y & y\end{array}\right]^{\mathrm{T}} \quad$ and $\quad \mathbf{q}=\left[q_{1}, q_{2}, \ldots, q_{n}, q_{n+1}, \ldots\right.$, $\left.q_{n+m}\right]^{\mathrm{T}}$ is the extended vector of generalized variables. Note that $\boldsymbol{\Gamma}$ may have more than one parameter and that $\xi(\mathbf{q})$ represents the set of all points inside and on the boundary to the swept volume. The uniqueness of this formulation is evidenced by its invariance under any subsequent revolve or extrude operation. Indeed, even when sweep parameters are changed, the resulting $\boldsymbol{\xi}(\mathbf{q})$ is concurrently updated without the need for reformulating the problem. In order to develop a systematic method for generating the solid, we employ a convention in affixing frames. Once frames are established, the transformation matrices ${ }^{(i-1)} \mathbf{T}_{i}$ will be determined.

\subsection{Convention for affixing frames}

In order to obtain a systematic method for generating a $(4 \times 4)$ homogeneous transformation matrix between any two sweeps, the convention for establishing coordinate systems introduced by Denavit and Hartenberg is adopted. The procedure for establishing triads is implemented as:

1. Name each operation starting with $1,2, \ldots, n$ in reverse order;

2. Embed the $\mathrm{z}_{i-1}$ axis along the $i$ th sweep axis;

3. Embed the $\mathbf{x}_{i}$ axis normal to the $\mathbf{z}_{i-1}$ axis and $\mathbf{y}_{i}=\mathbf{z}_{i} \times \mathbf{x}_{i}$;

4. Define the geometric entity to be swept with respect to the $n$th coordinate frame. The location of the origin of the first frame (frame 0) can be chosen to be anywhere along the $\mathbf{z}_{0}$ axis;

5 . Denote the entity variables as $q_{n+1}, \ldots, q_{n+m}$. For example, $\boldsymbol{\Gamma}(u, v)$ becomes $\boldsymbol{\Gamma}\left(q_{n+1}, q_{n+2}\right)$.

To illustrate this convention, consider revolving the surface $\boldsymbol{\Gamma}(u, v)$ shown in Fig. 6(a) about an axis $\mathbf{a}_{1}$ by an angle $\theta_{1}$. The resulting volume is extruded in the direction of the axis $\mathbf{a}_{2}$ by a distance $d_{2}$, followed by another revolving sweep about the axis $\mathbf{a}_{3}$ with an angle $\theta_{3}$. Revolving sweeps are replaced by revolute joints shown as cylindrical surfaces in Fig. 6(b) and located along the axes $\mathbf{a}_{1}$ and $\mathbf{a}_{3}$. Extrude sweeps are replaced by prismatic joints shown as prismatic members in Fig. 6(b). The $\mathbf{z}_{i}$ axes are now located along each joint, starting with the last sweep operation such that the axes are named $\mathbf{z}_{0}, \mathbf{z}_{1}$, and $\mathbf{z}_{2}$. The $\mathbf{x}$-axes are located using the second convention and the $\mathbf{y}$-axes are not shown.

\section{Implicit function theorem and varieties in the manifold}

Every point in the solid model characterized by $\boldsymbol{\xi}(\mathbf{q})$ is subject to the constraints of the parameters of Eq. (6). To 


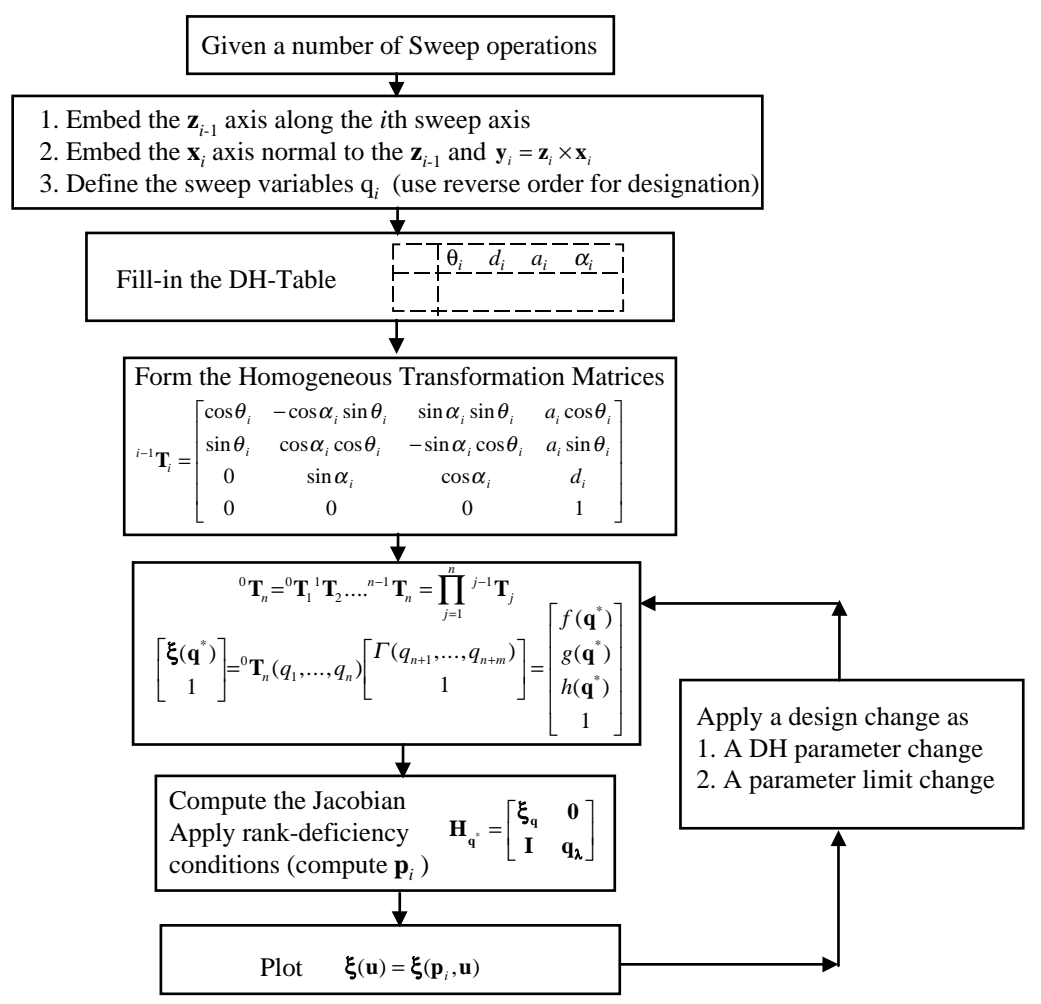

Fig. 7. Algorithm for creating and representing a solid model using the DH-method.

impose the inequality constraints of Eq. (2) in terms of the sweep variables, it is possible to transform the constraint of Eq. (2) into equality by introducing a new set of generalized coordinates $\lambda_{i}$ such that it is rewritten as

$q_{i}=a_{i}+b_{i} \sin \lambda_{i} i=1, \ldots, n+m$

where $a_{i}=\left(q_{i}^{\max }+q_{i}^{\min }\right) / 2$ and $b_{i}=\left(q_{i}^{\max }+q_{i}^{\min }\right) / 2$ are the mid-point and half-range of the constraint. Augmenting $\boldsymbol{\xi}(\mathbf{q})$ by the constraints, a point in the solid model is represented by a manifold as

$\mathbf{H}\left(\mathbf{q}^{*}\right)=\left[\begin{array}{c}\boldsymbol{\xi}(\mathbf{q}) \\ q_{i}-a_{i}-b_{i} \sin \lambda_{i}\end{array}\right] \quad i=1, \ldots, n+m$

where $\mathbf{q}^{*}=\left[\mathbf{q}^{\mathrm{T}} \boldsymbol{\lambda}^{\mathrm{T}}\right]^{\mathrm{T}}$ is $2(n+m) \times 1$ and is the vector of all generalized coordinates. The Jacobian of the constraint function is a nonsquare $(3+n+m) \times 2(n+m)$ matrix given by

$\mathbf{H}_{\mathbf{q}^{*}}=\left[\begin{array}{cc}\xi_{\mathbf{q}} & \mathbf{0} \\ \mathbf{I} & \mathbf{q}_{\boldsymbol{\lambda}}\end{array}\right]$

where the subscript notation denotes a derivative such that $\mathbf{H}_{\mathbf{q}^{*}}=\partial \mathbf{H} / \partial \mathbf{q}^{*}$, and $\xi_{\mathbf{q}}=\partial \boldsymbol{\xi} / \partial \mathbf{q}$, and $\mathbf{q}_{\boldsymbol{\lambda}}=\partial \mathbf{q} / \partial \boldsymbol{\lambda}$, and where $\mathbf{I}$ is the identity matrix. In order to have a wellposed formulation; constraints that are used to model the geometry of this problem should be independent, except at certain critical surfaces in the manifold (Implicit Function Theorem) when the Jacobian becomes singular. It is important, therefore, that there not be open sets in the space of the generalized parameters in which the constraints are redundant. Redundancy occurs when the Jacobian $\mathbf{H}_{\mathbf{q}}$, is rank-deficient which will subsequently define varieties in the manifold. For an $n$-parameter solid, $\mathbf{H}_{\mathbf{q}}$ is rank deficient on the basis of two conditions that were derived by [17] and are generalized in Appendix A. Singularities as a result of the Jacobian rank deficiency condition are denoted by $\mathbf{p}_{i}$. Varieties in the manifold are expressed by substituting the singularities $\mathbf{p}_{i}$ into $\boldsymbol{\xi}(\mathbf{q})$ such that the equation of a variety is

$\boldsymbol{\xi}(\mathbf{u})=\boldsymbol{\xi}\left(\mathbf{u}, \mathbf{p}_{i}\right)$

The algorithm for creating and representing a solid model that is as a result of sweep operations and based on the Denavit-Hartenberg representation is shown in Fig. 7.

\subsection{Mass properties}

Because this formulation yields closed form surface equations describing the boundary of the solid model, it is now possible to apply the divergence theorem to compute the various mass properties that are essential to a solid modeler. The contribution of a variety $\boldsymbol{\xi}^{(i)}(\mathbf{u})$, where $\mathbf{u}=\left[q_{1} q_{2}\right]^{\mathrm{T}}$, to the volume of a solid model with unit normal $\hat{\mathbf{n}}^{(i)}$ using the divergence theorem is given by

$3 V=\iint_{A} \boldsymbol{\xi}^{(i)}(\mathbf{u}) \cdot \hat{\mathbf{n}}^{(i)} \mathrm{d} A$ 
The area is given by $\mathrm{d} A=\left\|\boldsymbol{\xi}_{q_{1}}^{(i)} \times \boldsymbol{\xi}_{q_{2}}^{(i)}\right\| \mathrm{d} q_{1} \mathrm{~d} q_{2}$ and for a variety which is a parametric entity, the normal vector is written as

$\hat{\mathbf{n}}^{(i)}=\frac{\boldsymbol{\xi}_{q_{1}}^{(i)} \times \boldsymbol{\xi}_{q_{2}}^{(i)}}{\left\|\boldsymbol{\xi}_{q_{1}}^{(i)} \times \boldsymbol{\xi}_{q_{2}}^{(i)}\right\|}$

Substituting for $\mathrm{d} A$ and for $\hat{\mathbf{n}}^{(i)}$ into Eq. (13), the volume $V$ of a solid model enclosed by varieties is the summation of the contribution of all boundary varieties as (note that $\mathbf{n}$ is not a unit vector)

$V=\frac{1}{3} \sum_{i}^{\eta} \iint_{A} \xi^{(i)}\left(q_{1}, q_{2}\right) \cdot \mathbf{n}^{(i)} \mathrm{d} q_{1} \mathrm{~d} q_{2}$

Note that similarly, moments and products of inertia can be computed.

\section{Examples}

\subsection{An introductory example}

Consider revolving the curve $\boldsymbol{\Gamma}(t)$ about the axis $\mathbf{a}_{1}$ with an angle $\theta_{1}$ constrained by $\left(-\pi / 4<\theta_{1}<5 \pi / 4\right)$ as shown in Fig. 8(a), and where the curve is constrained as $(0<t<$ $3 \pi / 2$ ). The resulting surface will then be swept again about axis $\mathbf{a}_{2}$ shown in Fig. 8(b) with angle $\left(0<\theta_{2}<\pi / 2\right)$, followed by another sweep about axis $\mathbf{a}_{3}$, with an angle $\left(0<\theta_{3}<\pi\right)$.

Using the DH-representation method, the $\mathbf{z}_{i}$-axes are
Substituting values for each row of the DH-table into Eq. (1) yields the following matrices:

$$
\begin{aligned}
{ }^{0} \mathbf{T}_{1}\left(q_{1}\right) & =\left[\begin{array}{cccc}
\cos q_{1} & 0 & \sin q_{1} & 0 \\
\sin q_{1} & 0 & -\cos q_{1} & 0 \\
0 & 1 & 0 & 30 \\
0 & 0 & 0 & 1
\end{array}\right], \\
{ }^{1} \mathbf{T}_{2}\left(q_{2}\right) & =\left[\begin{array}{cccc}
\cos q_{2} & -\sin q_{2} & 0 & 20 \cos q_{2} \\
\sin q_{2} & \cos q_{2} & 0 & 20 \sin q_{2} \\
0 & 0 & 1 & 0 \\
0 & 0 & 0 & 1
\end{array}\right], \\
{ }^{2} \mathbf{T}_{3}\left(q_{3}\right) & =\left[\begin{array}{cccc}
\cos q_{3} & 0 & \sin q_{3} & 0 \\
\sin q_{3} & 0 & -\cos q_{3} & 0 \\
0 & 1 & 0 & 0 \\
0 & 0 & 0 & 1
\end{array}\right],
\end{aligned}
$$

and the swept curve written in terms of the third reference frame is $\boldsymbol{\Gamma}\left(q_{4}\right)=\left[5 \cos q_{4} 5 \sin q_{4} 7\right]^{\mathrm{T}}$. Performing the multiplication of Eq. (4) yields the set representing every point in the solid model as

$$
\left[\begin{array}{c}
\boldsymbol{\xi}(\mathbf{q}) \\
1
\end{array}\right]={ }^{0} \mathbf{T}_{1}\left(q_{1}\right)^{1} \mathbf{T}_{2}\left(q_{2}\right)^{2} \mathbf{T}_{3}\left(q_{3}\right)\left[\begin{array}{c}
\boldsymbol{\Gamma}\left(q_{4}\right) \\
1
\end{array}\right]
$$

where

$\xi(\mathbf{q})=$

$\left[\begin{array}{c}5 \cos q_{1} \cos q_{2} \cos q_{3} \cos q_{4}-5 \cos q_{1} \sin q_{2} \sin q_{3} \cos q_{4}+5 \sin q_{1} \sin q_{4}+7 \cos q_{1} \cos q_{2} \sin q_{3}+7 \cos q_{1} \sin q_{2} \cos q_{3}+20 \cos q_{1} \cos q_{2} \\ 5 \sin q_{1} \cos q_{2} \cos q_{3} \cos q_{4}-5 \sin q_{1} \sin q_{2} \sin q_{3} \cos q_{4}-5 \cos q_{1} \sin q_{4}+7 \sin q_{1} \cos q_{2} \sin q_{3}+7 \sin q_{1} \sin q_{2} \cos q_{3}+20 \sin q_{1} \cos q_{2} \\ 5 \sin q_{2} \cos q_{3} \cos q_{4}+5 \cos q_{2} \sin q_{3} \cos q_{4}+7 \sin q_{2} \sin q_{3}-7 \cos q_{2} \cos q_{3}+20 \sin q_{2}+30\end{array}\right]$

located along the sweep axes and are denoted by $\mathbf{z}_{0}, \mathbf{z}_{1}, \mathbf{z}_{2}$ where the numbering scheme has started from the last sweep operation. Note also that the sweep variables $\theta_{i}$ s are also changed to $q_{i}$ s but in reversed order where the parameter $t$ of the curve $\boldsymbol{\Gamma}$ is denoted last as $q_{4}$ such that $\mathbf{q}=\left[q_{1}, q_{2}, q_{3}, q_{4}\right]^{\mathrm{T}}=\left[\theta_{3}, \theta_{2}, \theta_{1}, t\right]^{\mathrm{T}}$. The $\mathbf{x}_{i}$ axes are also shown in Fig. 8(c) and are located subject to the rules of Section 2. Dimensions are shown in Fig. 8(c) and the DHparameters are entered into Table 1.

Table 1

DH-parameters of the sweep of example 1

\begin{tabular}{lllll}
\hline Joint & $\theta_{i}$ & $d_{i}$ & $a_{i}$ & $\alpha_{i}$ \\
\hline 1 & $q_{1}$ & 30 & 0 & $\pi / 2$ \\
2 & $q_{2}$ & 0 & 20 & 0 \\
3 & $q_{3}$ & 0 & 0 & $\pi / 2$
\end{tabular}

and the parameterization of the inequality constraints yields $q_{1}=\pi / 2+(\pi / 2) \sin \lambda_{1}, \quad q_{2}=\pi / 4+(\pi / 4) \sin \lambda_{2}, \quad q_{3}=$ $\pi / 2+(3 \pi / 4) \sin \lambda_{3}$, and $q_{4}=\pi / 2+(\pi / 2) \sin \lambda_{4}$. Using the Jacobian row-rank deficiency condition, there are four subJacobians, whose determinants are computed and set to zero as

$$
\begin{aligned}
& \operatorname{det}\left(\hbar_{1} \hbar_{2} \hbar_{3}\right)_{1}=1500 \cos q_{2} \cos q_{3} \\
& +1500 \cos q_{4} \cos q_{2}\left(\cos q_{3}\right)^{2}+2250\left(\cos q_{3}\right)^{2} \sin q_{2} \\
& -500 \cos q_{4} \cos q_{2} \sin q_{3}+2250 \cos q_{2} \sin q_{3} \cos q_{3} \\
& -1500 \cos q_{4} \sin q_{3} \cos q_{3} \sin q_{2}+250\left(\cos q_{4}\right)^{2} \sin q_{2} \\
& -250\left(\cos q_{4}\right)^{2} \cos q_{2} \cos q_{3} \sin q_{3}-750 \cos q_{2} \cos q_{4} \\
& -250\left(\cos q_{4}\right)^{2} \times\left(\cos q_{3}\right)^{2} \sin q_{2}=0
\end{aligned}
$$




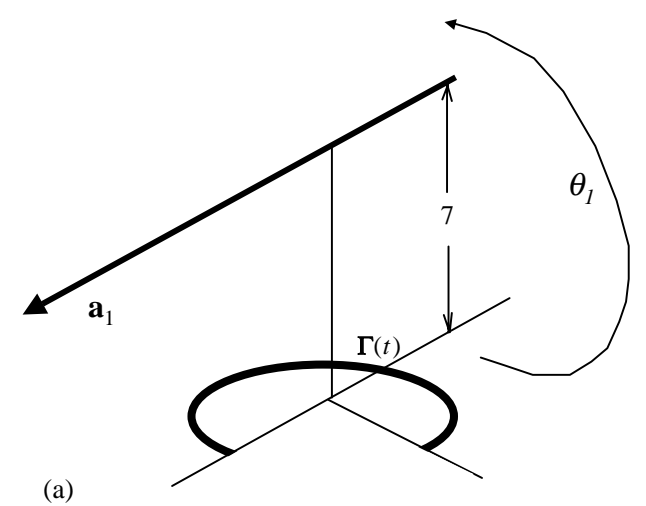

(a)

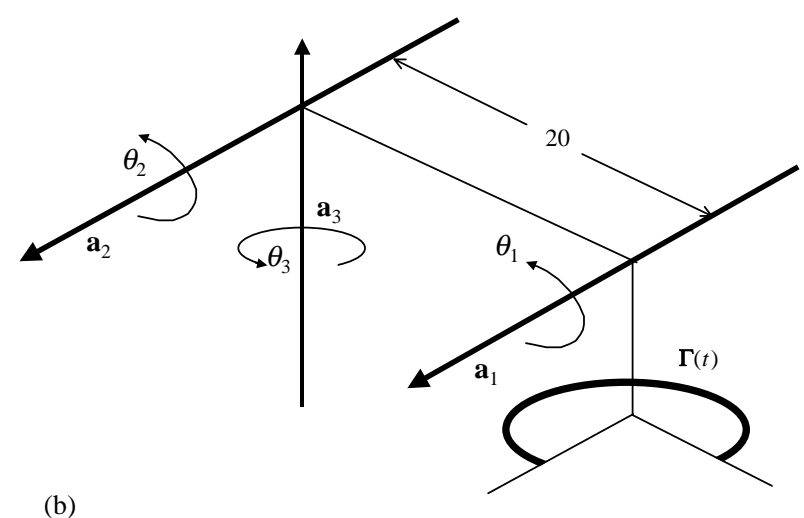

(b)

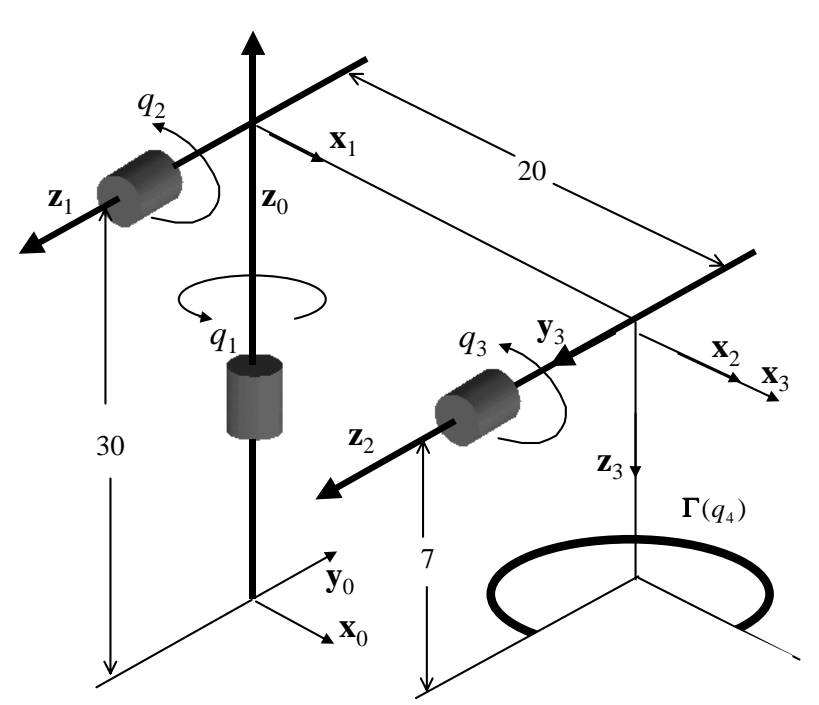

(c)

Fig. 8. (a) A sweep of a curve about an axis. (b) Consecutive sweeps of a curve. (c) The DH-representation of the complete sweep.

$\operatorname{det}\left(\hbar_{1} \hbar_{2} \hbar_{4}\right)_{2}=-500 \cos q_{2} \sin q_{4} \cos q_{3}$

$-750\left(\cos q_{3}\right)^{2} \sin q_{4} \sin q_{2}$

$-250 \sin q_{4} \cos q_{4} \cos q_{2}\left(\cos q_{3}\right)^{2}$

$-750 \cos q_{2} \sin q_{3} \sin q_{4} \cos q_{3}$

$+250 \cos q_{4} \sin q_{3} \sin q_{4} \cos q_{3} \sin q_{2}=0$ $\operatorname{det}\left(\hbar_{1} \hbar_{3} \hbar_{4}\right)_{3}=-250 \cos q_{2} \sin q_{4} \cos q_{4}=0$

$\operatorname{det}\left(\hbar_{2} \hbar_{3} \hbar_{4}\right)_{4}=250\left(\cos q_{4}\right)^{2} \sin q_{3}-750 \cos q_{4} \cos q_{3}=0$

Condition (i) singularities are the simultaneous solutions to 17(a)-(d)

$\mathbf{p}_{1}=\left\{q_{3}=\arctan (3), q_{4}=0\right\}$

$\mathrm{p}_{3}=\left\{q_{3}=\pi / 2, q_{4}=\pi / 2\right\}$

$\mathbf{p}_{2}=\left\{q_{3}=\pi-\arctan (3), q_{4}=\pi\right\}$

$p_{4}=\left\{q_{3}=\pi / 2, \quad q_{4}=3 \pi / 2\right\}$

Consider the limit $\partial \mathbf{q}^{\lim }=\left[q_{1}^{\max }=\pi\right]$, therefore, $\mathbf{w}=$ $\left[\begin{array}{lll}q_{2} & q_{3} & q_{4}\end{array}\right]^{\mathrm{T}}$. The Jacobian $\left[\xi_{\mathbf{q}} \oplus \xi_{q_{1}}\right]$ is computed and the rank deficiency condition yields $\hat{\mathbf{s}}=\left[q_{4}=\pi / 2\right]$. Therefore, the singular set is $\mathbf{p}_{31}=\left[\hat{\mathbf{s}} \cup \partial \mathbf{q}^{\mathrm{lim}}\right]=\left\{q_{1}=\pi, q_{4}=\right.$ $\pi / 2\}$. Similarly, other sets are computed as

$\mathbf{p}_{5}=\left\{q_{3}=-\pi / 4, q_{4}=0\right\}$,

$\mathbf{p}_{6}=\left\{q_{3}=-\pi / 4, q_{4}=\pi\right\}$,

$\mathbf{p}_{7}=\left\{q_{3}=5 \pi / 4, q_{4}=0\right\}$,

$\mathbf{p}_{8}=\left\{q_{3}=5 \pi / 4, \quad q_{4}=\pi\right\}$,

$\mathbf{p}_{17}=\left\{q_{2}=0, q_{4}=0\right\}$,

$\mathbf{p}_{18}=\left\{q_{2}=0, q_{4}=\pi\right\}$

$\mathbf{p}_{19}=\left\{q_{2}=0, q_{4}=\pi / 2\right\}$,

$\mathbf{p}_{20}=\left\{q_{2}=0, q_{4}=3 \pi / 2\right\}$,

$\mathbf{p}_{21}=\left\{q_{2}=\pi / 2, q_{4}=0\right\}$

$\mathbf{p}_{22}=\left\{q_{2}=\pi / 2, q_{4}=\pi\right\}$,

$\mathbf{p}_{23}=\left\{q_{2}=\pi / 2, q_{4}=\pi / 2\right\}$,

$\mathbf{p}_{24}=\left\{q_{2}=\pi / 2, q_{4}=3 \pi / 2\right\}$

$\mathbf{p}_{29}=\left\{q_{1}=0, q_{4}=\pi / 2\right\}$,

$\mathbf{p}_{30}=\left\{q_{1}=0, q_{4}=3 \pi / 2\right\}$, and

$\mathbf{p}_{32}=\left\{q_{1}=\pi, q_{4}=3 \pi / 2\right\}$

For $\operatorname{dim}\left[\partial \mathbf{q}^{\lim }\right]=n+m-2$, the following sets are identified.

$$
\begin{aligned}
& \mathbf{p}_{9}=\left\{q_{3}=-\pi / 4, q_{1}=0\right\}, \\
& \mathbf{p}_{10}=\left\{q_{3}=-\pi / 4, \quad q_{1}=\pi\right\}, \\
& \mathbf{p}_{11}=\left\{q_{3}=5 \pi / 4, \quad q_{1}=0\right\}
\end{aligned}
$$




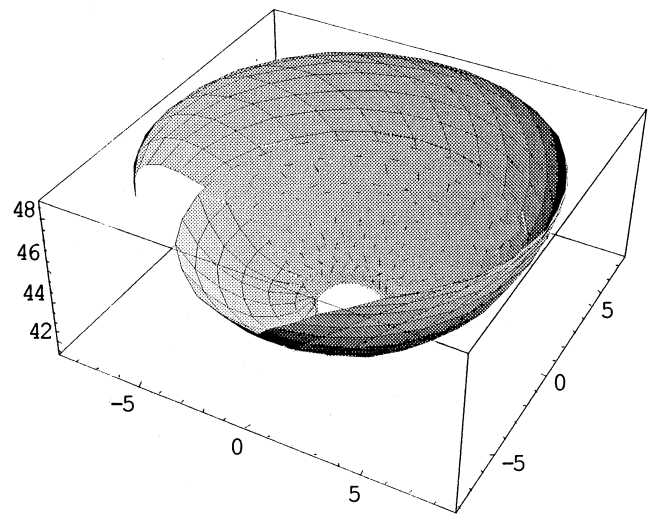

Fig. 9. Variety $\xi_{14}\left(\mathbf{p}_{14}, \mathbf{u}\right)$.

Table 2

DH-parameters for the arbitrary revolving about 4-axes

\begin{tabular}{llrll}
\hline Joint & $\theta_{I}$ & $d_{i}$ & $a_{i}$ & $\alpha_{I}$ \\
\hline 1 & $q_{1}$ & 10 & 10 & $\pi / 3$ \\
2 & $q_{2}$ & 8 & 12 & $\pi / 3$ \\
3 & $q_{3}$ & 16 & 20 & $\pi / 3$ \\
\hline
\end{tabular}

$$
\begin{aligned}
& \mathbf{p}_{12}=\left\{q_{3}=5 \pi / 4, \quad q_{1}=\pi\right\}, \\
& \mathbf{p}_{13}=\left\{q_{3}=-\pi / 4, q_{2}=0\right\}, \\
& \mathbf{p}_{14}=\left\{q_{3}=-\pi / 4, \quad q_{2}=\pi / 2\right\} \\
& \mathbf{p}_{15}=\left\{q_{3}=5 \pi / 4, \quad q_{2}=0\right\}, \\
& \mathbf{p}_{16}=\left\{q_{3}=5 \pi / 4, \quad q_{2}=\pi / 2\right\}, \\
& p_{25}=\left\{q_{2}=0, q_{1}=0\right\}, \\
& \mathbf{p}_{26}=\left\{q_{2}=0, q_{1}=5 \pi / 4\right\}, \\
& \mathbf{p}_{27}=\left\{q_{2}=\pi / 2, \quad q_{1}=0\right\}, \\
& \mathbf{p}_{28}=\left\{q_{2}=\pi / 2, \quad q_{1}=\pi\right\}
\end{aligned}
$$

Substituting each singular set into $\boldsymbol{\xi}(\mathbf{q})$ yields the equation of a variety. For example, substituting the set $\mathbf{p}_{14}=$ $\left\{q_{2}=\pi / 2, q_{3}=-\pi / 4\right\}$ into Eq. (24) yields the equation $\boldsymbol{\xi}_{14}\left(\mathbf{p}_{14}, \mathbf{u}\right)=\boldsymbol{\xi}_{14}(\mathbf{u})$

$$
=\left[\begin{array}{c}
5 \cos q_{1}+3.535 \cos q_{1} \cos q_{4}+5 \sin q_{1} \sin q_{4} \\
5 \sin q_{1}+3.535 \sin q_{1} \cos q_{4}-5 \cos q_{1} \sin q_{4} \\
45+3.535 \cos q_{4}
\end{array}\right]
$$

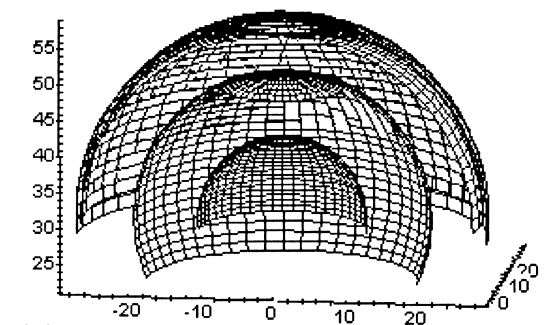

(a)

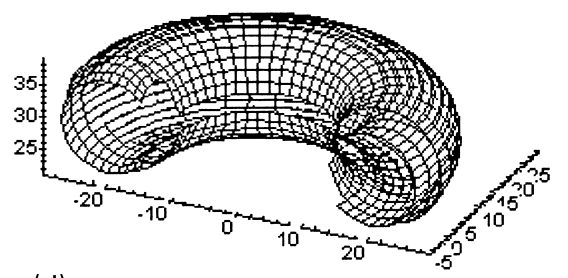

(d)

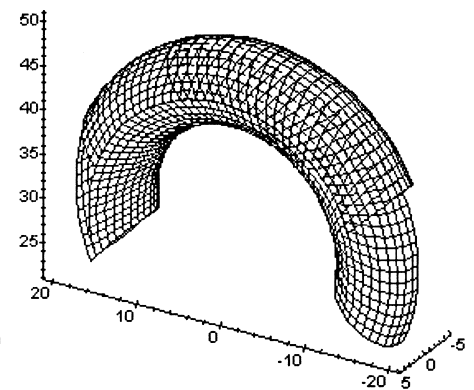

(b)

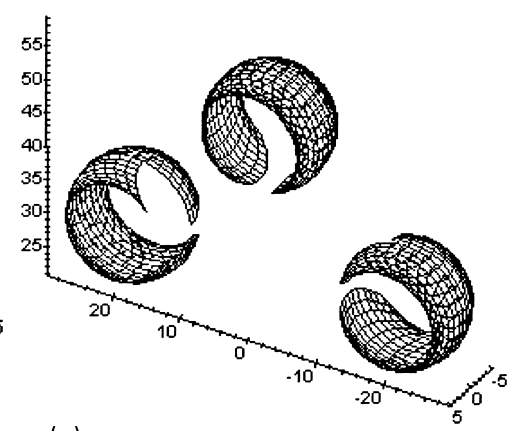

(e)

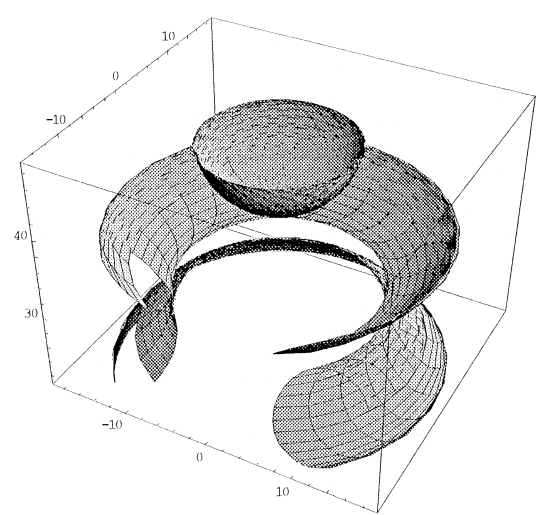

(c)

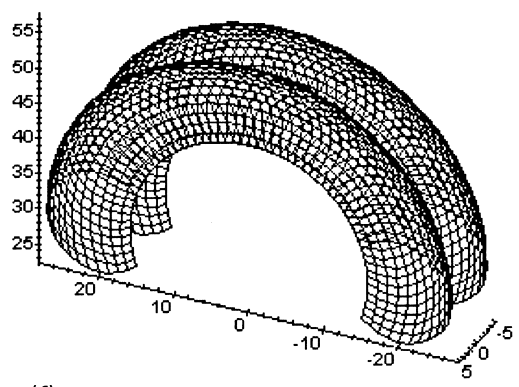

(f)

Fig. 10. (a) Varieties 1 through 8. (b) Varieties $\xi_{13}, \ldots, \xi_{16}$. (c) Varieties $\xi_{9}, \ldots, \xi_{12}$. (d) Varieties $\xi_{17}, \ldots, \xi_{20}$. (e) Varieties $\xi_{25}, \ldots, \xi_{28}$. (f) Varieties $\xi_{29}, \ldots, \xi_{32}$. 


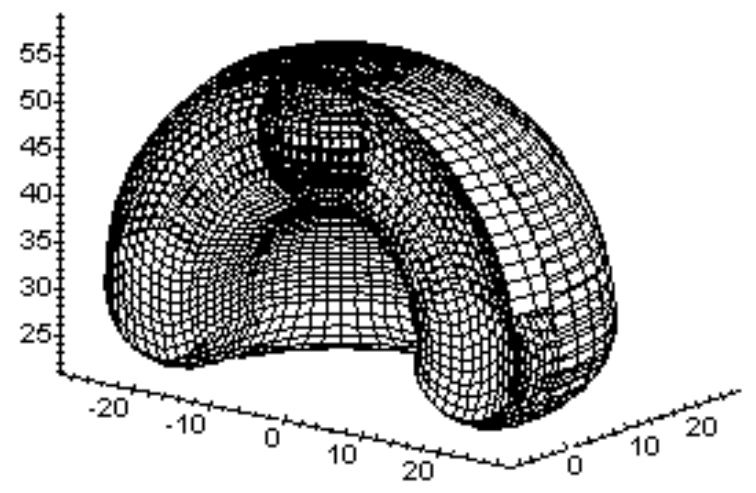

Fig. 11. The resulting solid model.

where $\mathbf{u}=\left[\begin{array}{ll}q_{1} & q_{4}\end{array}\right]^{\mathrm{T}}$. This variety is plotted and shown in Fig. 9.Varieties $\xi_{1}, \ldots, \xi_{8}$ are shown in Fig. 10(a); varieties $\xi_{9}, \ldots, \xi_{12}$ in Fig. 10(b); varieties $\xi_{13}, \ldots, \xi_{16}$ in Fig. 10(c); varieties $\xi_{17}, \ldots, \xi_{20}$ in Fig. $10(\mathrm{~d})$; varieties $\xi_{25}, \ldots, \xi_{28}$ in Fig. $10(\mathrm{e})$; and varieties $\xi_{29}, \ldots, \xi_{32}$ in Fig. 10(f). Combining all varieties, the solid model is shown in Fig. 11.

\subsection{Arbitrary revolving about four axes}

Consider the creation of a solid model by arbitrarily revolving the curve $\boldsymbol{\Gamma}\left(q_{4}\right)$ about four axes as shown in Fig. 12. The curve is given by

$\boldsymbol{\Gamma}\left(q_{4}\right)=\left[\begin{array}{c}3 \cos q_{4}+0.866 \sin q_{4} \\ 3 \sin q_{4}-0.866 \cos q_{4} \\ 3.5\end{array}\right] ;-\pi / 2<q_{4}<\pi / 2$

and is to be revolved first about $\mathbf{z}_{3}$, the result of which is revolved about $\mathbf{z}_{2}$, and subsequently revolving the result about $\mathbf{z}_{1}$. In this case, the four sweep axes are selected as general as possible with no intersections and in various directions. The $\mathbf{z}$ - and $\mathbf{x}$-axes are located as shown in Fig. 11 and the DH-parameters are presented in Table 2.

Substituting each row into the matrix of Eq. (1) yields

$$
\begin{aligned}
{ }^{0} \mathbf{T}_{1} & =\left[\begin{array}{cccc}
\cos q_{1} & -0.5 \sin q_{1} & 0.866 \sin q_{1} & 10 \cos q_{1} \\
\sin q_{1} & 0.5 \cos q_{1} & -0.866 \cos q_{1} & 10 \sin q_{1} \\
0 & 0.866 & 0.5 & 10 \\
0 & 0 & 0 & 1
\end{array}\right] \\
{ }^{1} \mathbf{T}_{2} & =\left[\begin{array}{cccc}
\cos q_{2} & -0.5 \sin q_{2} & 0.866 \sin q_{2} & 12 \cos q_{1} \\
\sin q_{2} & 0.5 \cos q_{2} & -.866 \cos q_{2} & 12 \sin q_{1} \\
0 & 0.866 & 0.5 & 8 \\
0 & 0 & 0 & 1
\end{array}\right] \\
{ }^{2} \mathbf{T}_{3} & {\left[\begin{array}{cccc}
\cos q_{3} & -0.5 \sin q_{3} & 0.866 \sin q_{3} & 20 \cos q_{3} \\
\sin q_{3} & 0.5 \cos q_{3} & -0.866 \cos q_{3} & 20 \sin q_{3} \\
0 & 0.866 & 0.5 & 16 \\
0 & 0 & 0 & 1
\end{array}\right] }
\end{aligned}
$$

and the vector characterizing the sweep is $\xi(q)$ where $\mathbf{q}=$ $\left[\begin{array}{llll}q_{1} & q_{2} & q_{3} & q_{4}\end{array}\right]^{\mathrm{T}}$

$\left[\begin{array}{c}\boldsymbol{\xi}(\mathbf{q}) \\ 1\end{array}\right]={ }^{0} \mathbf{T}_{1}\left(q_{1}\right)^{1} \mathbf{T}_{2}\left(q_{2}\right)^{2} \mathbf{T}_{3}\left(q_{3}\right)\left[\begin{array}{c}\boldsymbol{\Gamma}\left(q_{4}\right) \\ 1\end{array}\right]$

with the following constraints $-\pi / 2 \leq q_{1} \leq \pi / 2$, $-\pi / 2 \leq q_{2} \leq 2 \pi / 3$, and $-\pi / 2 \leq q_{3} \leq \pi / 2$.

Varieties are computed by substituting the singular sets into $\boldsymbol{\xi}(\mathbf{q})$ and are plotted in Fig. 13(a). A cross-section of the volume is taken at $z=0.8$ and depicted in Fig. 13(b).

\subsection{Design variation}

Consider revolving of the surface $\boldsymbol{\Gamma}(u, v)$ about the axis

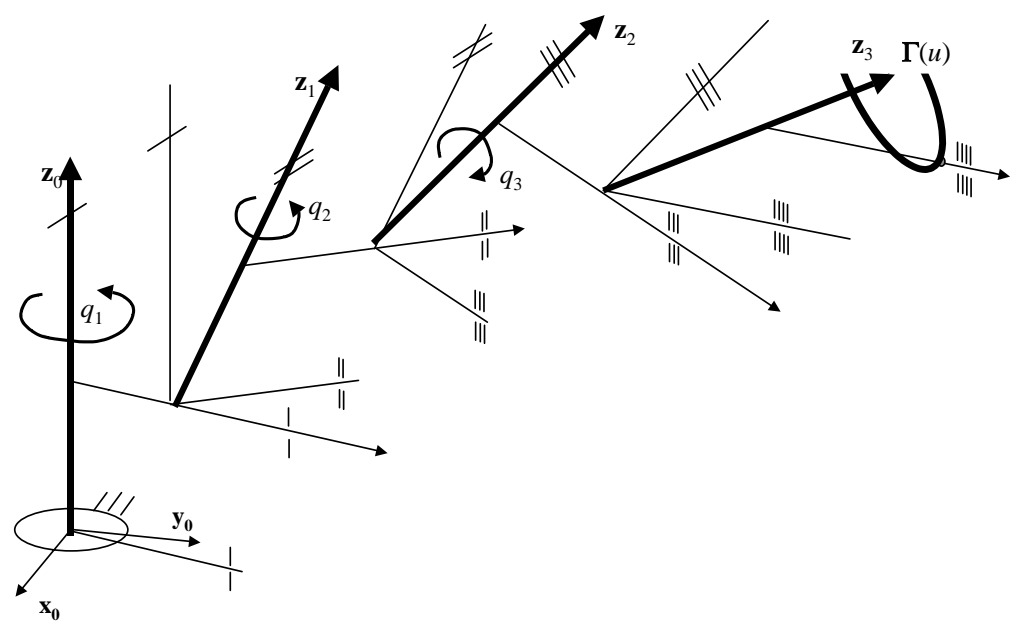

Fig. 12. A general four-parameter sweep of a curve. 


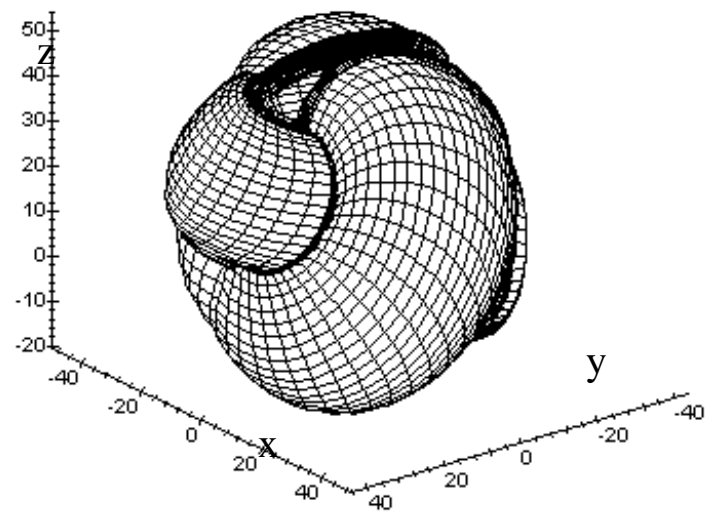

(a)

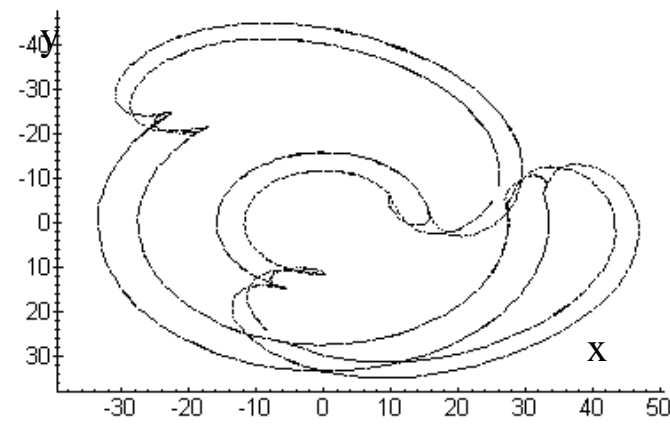

(b)

Fig. 13. (a) Solid model of the four-axes sweep. (b) A cross-section of the volume.

$\mathbf{z}_{2}$, subsequently extruding along the axis $\mathbf{z}_{1}$, and again along the axis $\mathbf{z}_{0}$ as shown Fig. 14, where the surface is given by

$\boldsymbol{\Gamma}(u, v)=\left[\begin{array}{lll}5 \cos v & 5+u & 5 \sin v\end{array}\right]^{\mathrm{T}} ;$

$5<u<10$, and $0<v<\pi / 2$

Using the DH representation method, the $\mathbf{x}$-axes are located as shown.

The homogenous transformation matrices are

$$
\begin{aligned}
{ }^{0} \mathbf{T}_{1} & =\left[\begin{array}{cccc}
1 & 0 & 0 & 0 \\
0 & 0 & -1 & 0 \\
0 & 1 & 0 & q_{1}+10 \\
0 & 0 & 0 & 1
\end{array}\right], \\
{ }^{1} \mathbf{T}_{2} & =\left[\begin{array}{cccc}
1 & 0 & 0 & 0 \\
0 & 0 & 1 & 0 \\
0 & -1 & 0 & q_{2}+7 \\
0 & 0 & 0 & 1
\end{array}\right], \text { and }{ }^{2} \mathbf{T}_{3} \\
& =\left[\begin{array}{cccc}
\sin q_{3} & 0 & \cos q_{3} & 0 \\
-\cos q_{3} & 0 & \sin q_{3} & 0 \\
0 & -1 & 0 & 0 \\
0 & 0 & 0 & 1
\end{array}\right]
\end{aligned}
$$

Exchanging $u$ and $v$ with $q_{4}$ and $q_{5}$, the vector $\boldsymbol{\xi}(\mathbf{q})$ characterizing every point model is

$$
\left[\begin{array}{c}
\boldsymbol{\xi}(\mathbf{q}) \\
1
\end{array}\right]={ }^{0} \mathbf{T}_{1}\left(q_{1}\right){ }^{1} \mathbf{T}_{2}\left(q_{2}\right)^{2} \mathbf{T}_{3}\left(q_{3}\right)\left[\begin{array}{c}
\boldsymbol{\Gamma}\left(q_{4}, q_{5}\right) \\
\mathbf{1}
\end{array}\right]
$$

with $10 \leq q_{1} \leq 20,7 \leq q_{2} \leq 15$, and $-\pi / 2 \leq q_{3} \leq \pi / 2$. As this is a five-parameter sweep, the resulting Jacbian is $(3 \times 5)$, hence $n=5$. A total of 90 singular sets exist. For example, varieties due to the boundary singular behavior produced by the limits of $q_{1}, q_{3}, q_{4}$ are shown in Fig. 15 . The complete swept volume of the five-parameter sweep is shown in Fig. 16.

Because of the unique properties of the $\mathrm{DH}$ representation method, variational effects due to design changes can be addressed. Consider for example introducing a change in the upper parametric limits of the generalized coordinates. A change in the upper limit of $q_{3}$ from $\pi / 2$ to $\pi / 4$; a change in the upper limit of $q_{4}$ from 10 to 8 ; and a change in the upper limit of $q_{5}$ from $\pi / 2$ to $\pi / 4$. The solid model can be readily updated and shown in Fig. 17(a). Furthermore, it is now possible to introduce changes in the design variables characterized by the location and orientation of the coordinate systems. For example, consider the change in the angle of the surface $\Gamma$ by introducing a change in the DH-Table (a tilt of $\pi / 4$ ). The homogeneous transformation matrices ${ }^{(i-1)} \mathbf{T}_{i}$ are updated accordingly, and the expression for $\boldsymbol{\xi}(\mathbf{q})$ is computed. The updated solid is now shown in Fig. 17(b).

\subsection{Mass properties}

In order to demonstrate the computation of the volume or a solid model that is a result of the DH representation, consider the sweep of a curve about an axis followed by

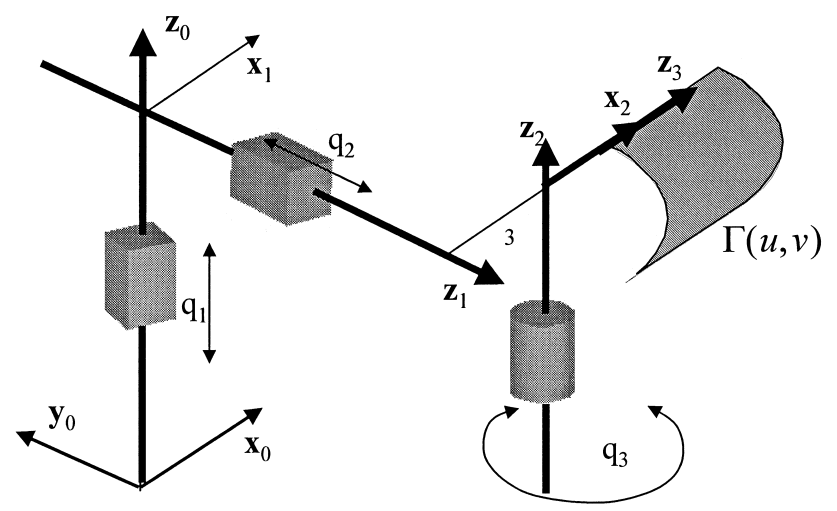

Fig. 14. Multiple sweeps of a surface to generate a five-parameter swept volume. 

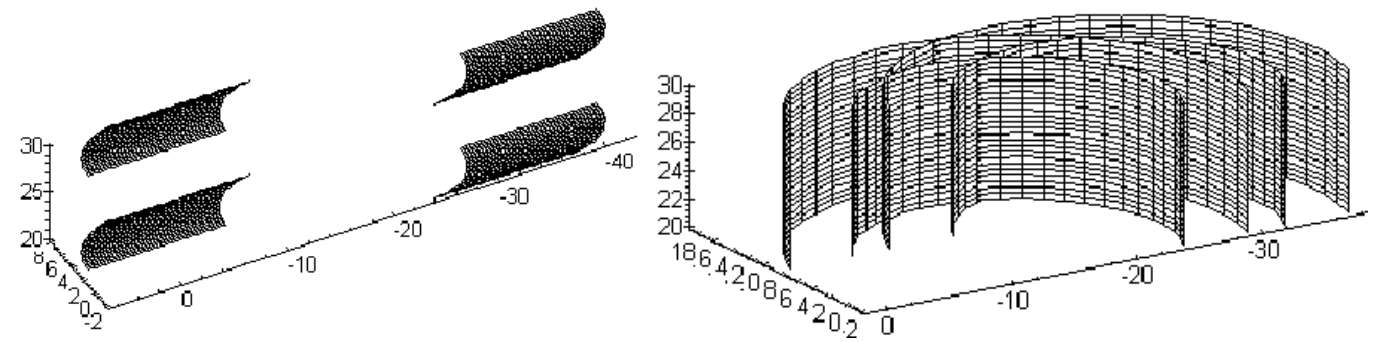

Fig. 15. Varieties of the manifold.

another sweep about a second axis as shown in Fig. 18, where the parameters are constrained as follows: $0<q_{1}<$ $270^{\circ},-120^{\circ}<q_{2}<120^{\circ}$, and $-120^{\circ}<q_{3}<120^{\circ}$.

Using the $\mathrm{DH}$ parameters, the manifold is characterized by

\subsection{A four parameter solid model}

The Denavit-Hartenberg parameters are presented in Table 2 and the resulting swept volume shown in Fig. 20.

$\xi(\mathbf{q})=\left[\begin{array}{c}10 \cos q_{1} \cos q_{2}+5 \cos q_{1} \cos q_{2} \cos q_{3}-5 \cos q_{1} \sin q_{2} \sin q_{3} \\ 10 \cos q_{2} \sin q_{1}+5 \cos q_{2} \cos q_{3} \sin q_{1}-5 \sin q_{1} \sin q_{2} \sin q_{3} \\ 10 \sin q_{2}+5 \cos q_{3} \sin q_{2}+5 \cos q_{2} \sin q_{3}\end{array}\right]$

Combining all varieties $\quad\left(\mathbf{p}_{1}=\left\{q_{3}=0\right\}\right.$, $\mathbf{p}_{2}=\left\{q_{3}=120^{\circ}\right\}, \quad \mathbf{p}_{3}=\left\{q_{3}=-120^{\circ}\right\} \quad \mathbf{p}_{4}=\left\{q_{1}=0\right\}$, $\mathbf{p}_{5}=\left\{q_{1}=270^{\circ}\right\}, \quad \mathbf{p}_{6}=\left\{q_{2}=-120^{\circ}\right\} \quad$ and $\left.\mathbf{p}_{7}=\left\{q_{2}=120^{\circ}\right\}\right)$, singularities $q_{2}=-120^{\circ}$ and $120^{\circ}$ yields the solid model shown in Fig. 18.

Boundary variety patches of the solid model are presented in Table 1.

The volume of this solid model is computed as 12484.3. To verify the results, cross-sectional cuts through the solid were introduced as shown in Fig. 19.

To validate the volume computation, a cross-section was computed at each unit along the $z$-axis, the area computed, and the volume calculated by integrating across the crosssections.

\begin{tabular}{llc}
\hline Variety & Parametric limits & $\begin{array}{l}\text { Volume } \\
\text { contribution }\end{array}$ \\
\hline$\xi^{(1)}$ & $0 \leq q_{1} \leq 3 \pi / 2 ;-2 \pi / 3 \leq q_{2} \leq 2 \pi / 3$ & 27547.1 \\
$\xi^{(2)}$ & $0 \leq q_{1} \leq 3 \pi / 2 ;-2 \pi / 3 \leq q_{2} \leq-\pi / 6$ & 3060.79 \\
$\xi^{(2)}$ & $\pi / 2 \leq q_{1} \leq \pi ; 2.68 \pi / 8 \leq q_{2} \leq 2 \pi / 3$ & 510.11 \\
$\xi^{(3)}$ & $0 \leq q_{1} \leq 3 \pi / 2 ; \pi / 6 \leq q_{2} \leq 2 \pi / 3$ & 3060.79 \\
$\xi^{(3)}$ & $\pi / 2 \leq q_{1} \leq \pi ;-2 \pi / 3 \leq q_{2} \leq-2.68 \pi / 8$ & 610.11 \\
$\xi^{(4)}$ & $-\pi / 3 \leq q_{2} \leq \pi / 3 ;-2 \pi / 3 \leq q_{3} \leq 2 \pi / 3$ & 0 \\
$\xi^{(5)}$ & $-\pi / 3 \leq q_{2} \leq \pi / 3 ;-2 \pi / 3 \leq q_{3} \leq 2 \pi / 3$ & 0 \\
$\xi^{(6)}$ & $\pi / 2 \leq q_{2} \leq \pi ;-2 \pi / 3 \leq q_{3} \leq 0$ & 1382.5 \\
$\xi^{(7)}$ & $\pi / 2 \leq q_{2} \leq \pi ; 0 \leq q_{3} \leq 2 \pi / 3$ & 1382.5 \\
& Total & 37453 \\
\hline & &
\end{tabular}

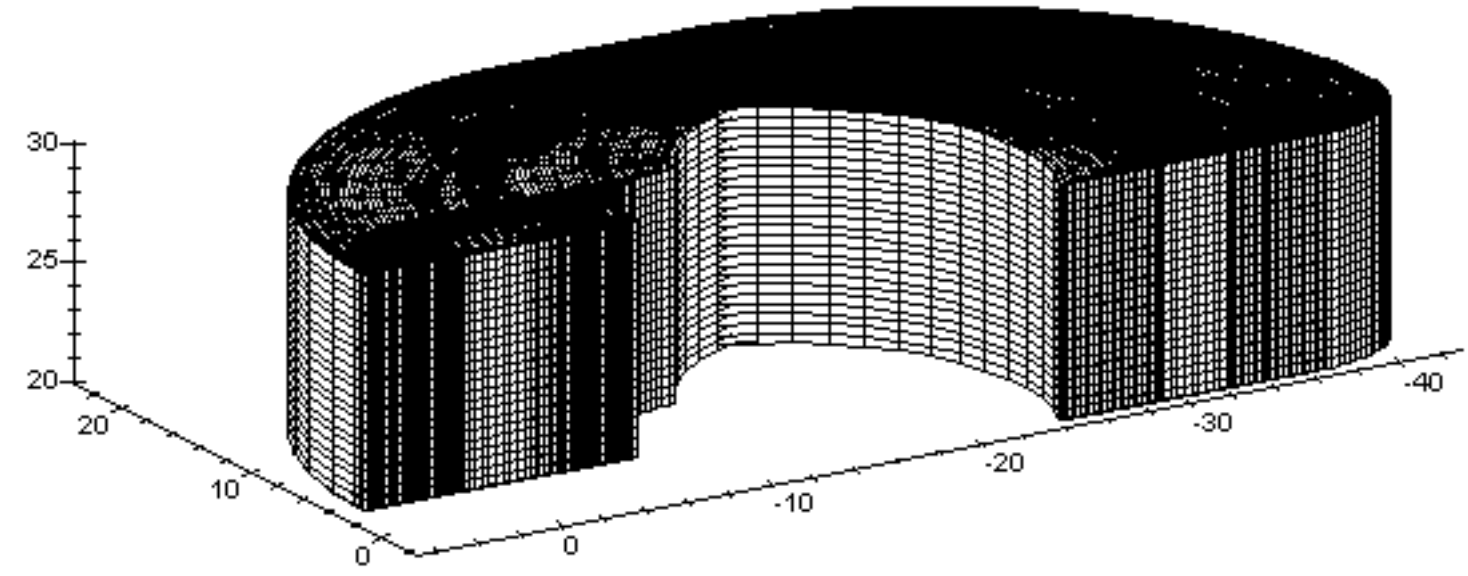

Fig. 16. Swept volume of the five-parameter sweep. 


\subsection{A five-parameter solid model}

Consider the sweep of the hemispherical surface given by $\boldsymbol{\Gamma}\left(q_{4}, q_{5}\right)=\left[-5 \cos q_{4} \sin q_{5}-5 \sin q_{4} \sin q_{5} 5 \cos q_{5}\right]^{\mathrm{T}}$; $0 \leq q_{4} \leq 2 \pi$ and $-\pi / 2 \leq q_{5} \leq \pi / 2$ three times as given by the following transformation matrices.

$$
{ }^{0} \mathrm{~T}_{1}=\left[\begin{array}{cccc}
\cos q_{1} & 0 & -\sin q_{1} & 0 \\
\sin q_{1} & 0 & \cos q_{1} & 0 \\
0 & -1 & 0 & 25 \\
0 & 0 & 0 & 1
\end{array}\right],
$$$$
{ }^{1} \mathbf{T}_{2}=\left[\begin{array}{cccc}
\sin q_{2} & 0 & \cos q_{2} & 0 \\
-\cos q_{1} & 0 & \sin q_{2} & 0 \\
0 & -1 & 0 & 0 \\
0 & 0 & 0 & 1
\end{array}\right] \text {, }
$$$$
{ }^{2} \mathbf{T}_{3}=\left[\begin{array}{cccc}
\sin q_{2} & -1 & 0 & 0 \\
1 & 0 & 0 & 0 \\
0 & 0 & 1 & 15+q_{3} \\
0 & 0 & 0 & 1
\end{array}\right]
$$

\begin{tabular}{llllc}
\hline Joint No. & $\theta$ & $d$ & $A$ & $\alpha$ \\
\hline 1 & $q 1-90$ & 5 & 0 & 90 \\
2 & $q 2+90$ & 0 & 0 & 90 \\
3 & 0 & $q 3+4$ & 0 & -90 \\
4 & $q 4$ & 0 & 0 & 90 \\
\hline
\end{tabular}

Rank deficiency criteria are applied to $\xi$ yielding the following singular sets.

$$
\begin{aligned}
& \mathbf{p}_{1}:\left\{q_{2}=\pi / 2, q_{4}=0, \text { and } q_{5}=\pi / 2\right\} \\
& \mathbf{p}_{2}:\left\{q_{2}=-\pi / 4, q_{4}=0, \text { and } q_{5}=0\right\}, \\
& \mathbf{p}_{3}:\left\{q_{2}=-\pi / 4, q_{4}=\pi / 2, \text { and } q_{5}=\pi / 2\right\} \\
& \mathbf{p}_{4}:\left\{q_{2}=-\pi / 4, q_{4}=3 \pi / 2, \text { and } q_{5}=\pi / 2\right\} \\
& \mathbf{p}_{5}:\left\{q_{3}=0, q_{4}=0, \text { and } q_{5}=0\right\}
\end{aligned}
$$

Unilateral constraints are defined as $0 \leq q_{1} \leq 2 \pi$, $-\pi / 4 \leq q_{2} \leq 5 \pi / 4,0 \leq q_{3} \leq 5$. Using Denavit-Harten$\mathbf{p}_{6}:\left\{q_{2}=\pi / 2, q_{3}=0\right.$, and $\left.q_{4}=0\right\}$, berg, the sweep is given by

$\boldsymbol{\xi}\left(\mathbf{q}^{*}\right)=\left[\begin{array}{c}-5 \sin q_{5} \cos q_{1} \sin q_{2} \sin q_{4}-5 \sin q_{5} \sin q_{1} \cos q_{4}+5 \cos q_{1} \cos q_{2} \cos q_{5}+15 \cos q_{1} \cos q_{2}+q_{3} \cos q_{1} \cos q_{2} \\ -5 \sin q_{5} \sin q_{1} \sin q_{2} \sin q_{4}+5 \sin q_{5} \cos q_{1} \cos q_{4}+5 \sin q_{1} \cos q_{2} \cos q_{5}+15 \sin q_{1} \cos q_{2}+q_{3} \sin q_{1} \cos q_{2} \\ 5 \cos q_{2} \sin q_{4} \sin q_{5}+5 \sin q_{2} \cos q_{5}+15 \sin q_{2}+q_{3} \sin q_{2}+25\end{array}\right]$

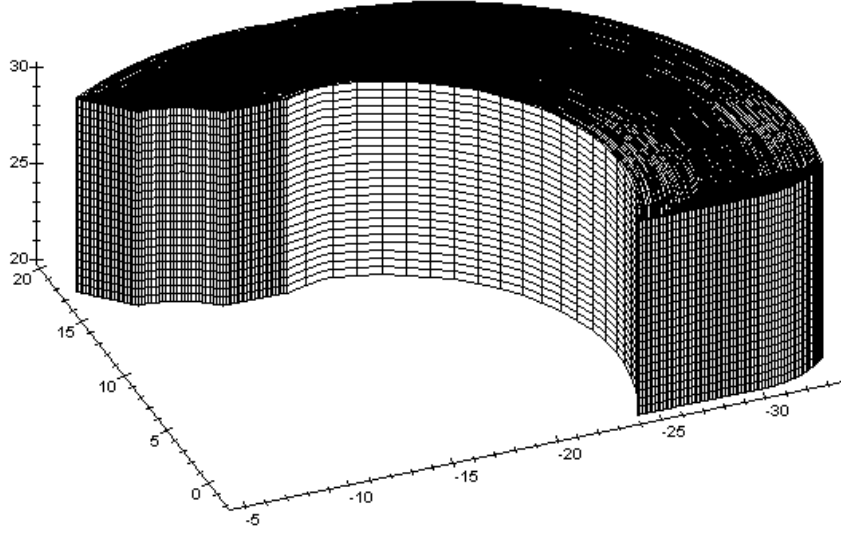

(a)

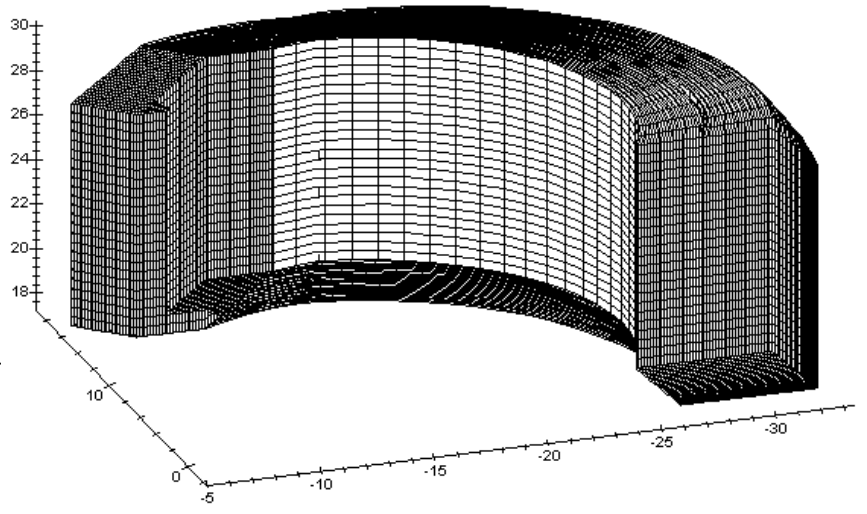

(b)

Fig. 17. (a) The updated part due to a design variation. (b) Effect of changing the DH-parameters. 
(a)

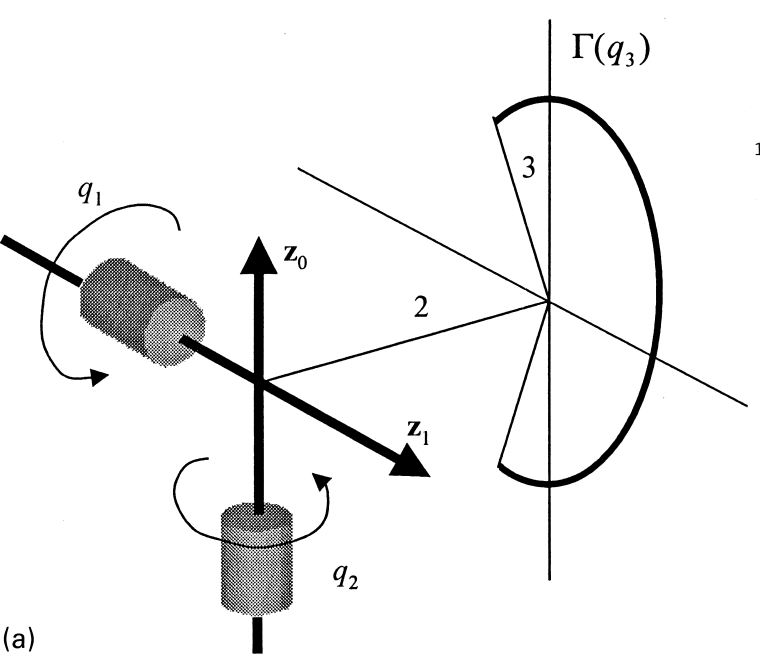

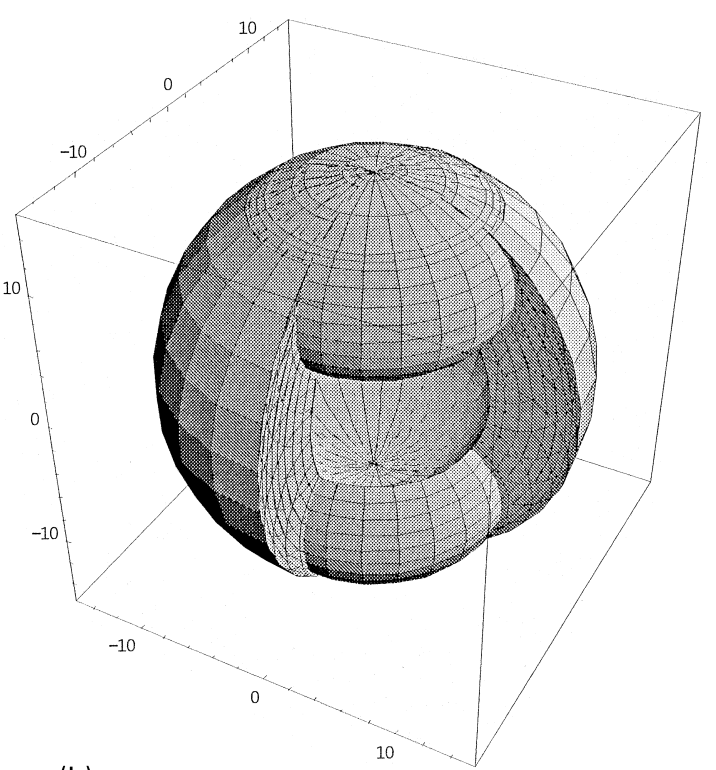

(b)

Fig. 18. (a) Revolving of a curve about two axes. (b) Resulting solid model.

$\mathbf{p}_{7}:\left\{q_{3}=5, q_{4}=0\right.$, and $\left.q_{5}=0\right\}$

$\mathbf{p}_{8}:\left\{q_{2}=\pi / 2, q_{3}=5\right.$, and $\left.q_{4}=0\right\}$,

$\mathbf{p}_{9}:\left\{q_{2}=-\pi / 4, q_{3}=0\right.$, and $\left.q_{4}=\pi / 2\right\}$

$p_{10}:\left\{q_{2}=-\pi / 4, q_{3}=5\right.$, and $\left.q_{4}=\pi / 2\right\}$

$\mathbf{p}_{11}:\left\{q_{3}=0\right.$ and $\left.q_{5}=\pi / 2\right\}$

$\mathbf{p}_{12}:\left\{q_{3}=5\right.$ and $\left.q_{5}=\pi / 2\right\}$
Note that hypersurfaces due to $\mathbf{p}_{11}$ and $\mathbf{p}_{12}$ are parametrized in three variables $\left(q_{1}, q_{2}\right.$, and $\left.q_{4}\right)$ indicating coupled singular behavior. A cross section of the workspace of the 5-DOF manipulator depicting all singular surfaces is shown in Fig. 21.

\section{Conclusions}

A mathematical formulation and a representation method are demonstrated for the creation of solid models. It was shown that the Denavit-Hartenberg representation method adopted from kinematics is well suited for

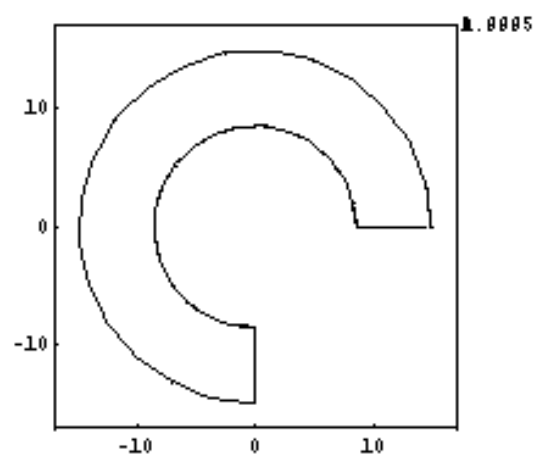

(a)

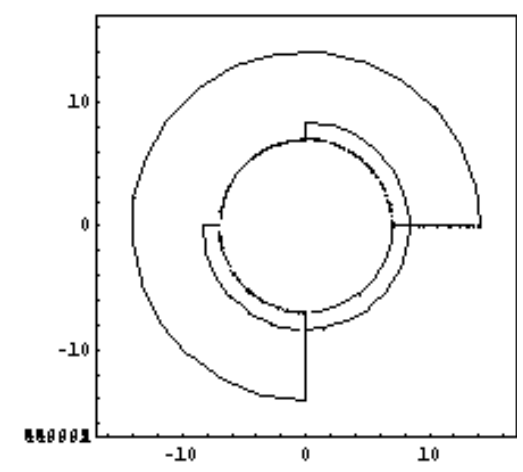

(b)

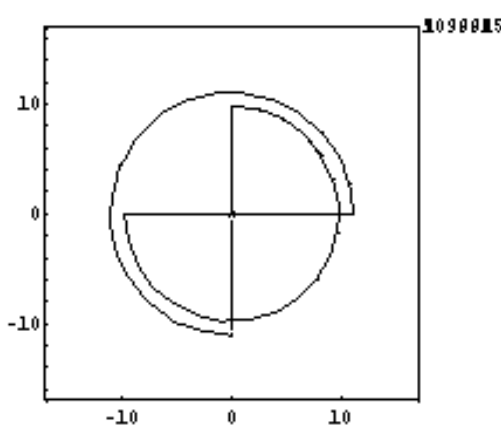

(c)

Fig. 19. (a) At $z=1.0$. (b) At $z=5.0$. (c) At $z=10.0$. 


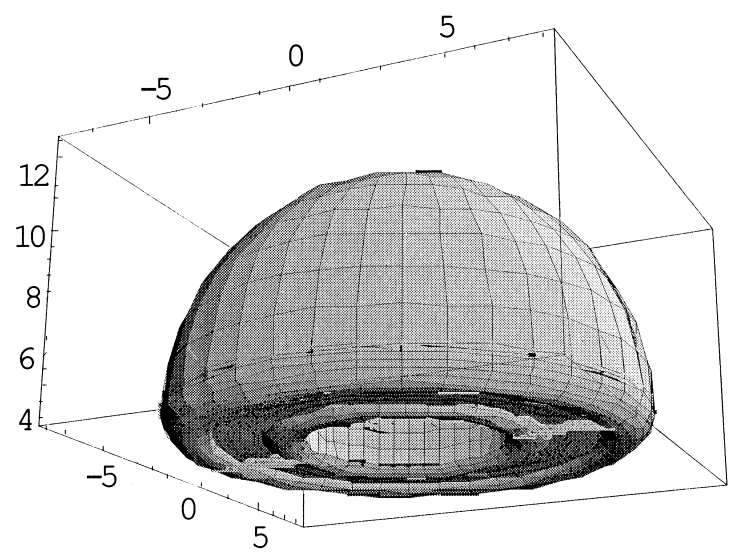

Fig. 20. The solid model.

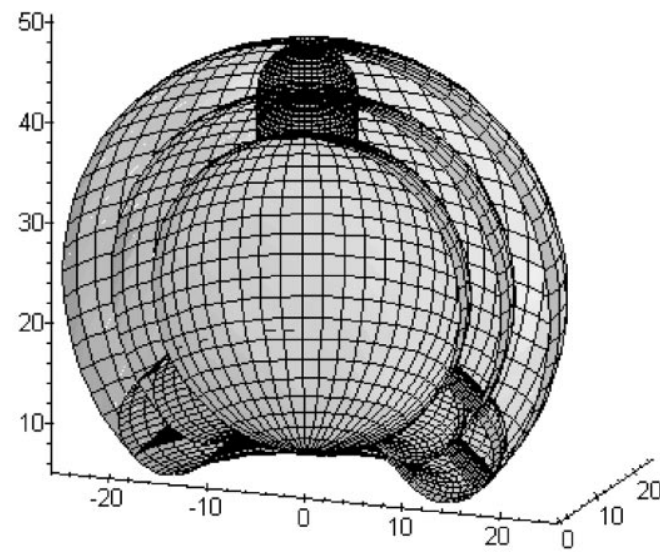

Fig. 21. Swept volume. the representation of solid models that are created as a result of multiple of sweep (combination of revolve and extrude) operations.

In CAD systems, it is often the case that a designer starts with a simple geometric entity and applies a number of consecutive sweeps to produce a desired shape. While various transformation vectors can be used to represent the resulting set, the $\mathrm{DH}$ representation method permits the designer to study a design change corresponding to a variation in one of the parameters. As a solid modeling method, the DH formulation can be used as a preprocessor to the sweep formulation in a CAD environment. An experimental computer code using Mathematica ${ }^{\circledR}$ was implemented for demonstration purposes but has been shown to have highpotential for implementation into CAD/CAM software.

In this formulation, it was shown that consecutive sweeps are systematically modeled using the DH representation method. The DH method relates any two such sweeps by only four parameters (four basic transformation matrices). This minimal representation makes it easier for addressing multiple sweeps and simplifies the computations. Parameter limits imposed in terms of inequality constraints are also taken into consideration where inequalities are converted to equalities through the introduction of slack variables. It was shown that the implicit function theorem applied to the non-square Jacobian delineate two types of singular sets. It was also shown that these sets when substituted into the position vector characterize equations of varieties of the manifold. Because of the closed-form equations describing the boundary of the solid model, it is possible to compute mass properties using the Divergence theorem. While this formulation does not replace existing methods of solid modeling, it is a preprocessor to a solid modeler that has the capability for generating solids from sweep operations.

\section{Appendix}

For a given non-square $(\mathbf{3}+\boldsymbol{n}+\boldsymbol{m}) \times \mathbf{2}(\boldsymbol{n}+\boldsymbol{m})$ Jacobian defined as

$\mathbf{H}_{\mathbf{q}^{*}}=\left[\begin{array}{cc}\xi_{\mathbf{q}} & \mathbf{0} \\ \mathbf{I} & \mathbf{q}_{\lambda}\end{array}\right]$

There exist two conditions for determining varieties of the manifold $\mathbf{H}$.

1. The upper left $(3 \times(n+m))$ corner matrix of $\mathbf{H}_{\mathbf{q}^{*}}$ is row rank deficient, i.e. $\operatorname{Rank}\left(\boldsymbol{\xi}_{\mathbf{q}}\right)<3$, i.e. $k=1$, 2 , or 3 . Solutions of the resulting $\eta$ equations of all three conditions are sets of constant generalized coordinates denoted by $\mathbf{p}_{i}$ and are characterized by the following set

$$
\begin{aligned}
\mathbf{p}_{i} & =\left\{\left[\begin{array}{c}
\operatorname{det}\left(\hbar_{i} \hbar_{j} \hbar_{k}\right)_{1} \\
\vdots \\
\operatorname{det}\left(\hbar_{i} \hbar_{j} \hbar_{k}\right)_{\eta}
\end{array}\right]=\mathbf{0}, \text { for } i, j, k\right. \\
& =1 \ldots(n+m) \text { and } i \neq j \neq k\} i=1,2, \ldots, \beta
\end{aligned}
$$

where $\hbar_{i}$ denotes a column of the matrix $\xi_{\mathbf{q}}=$ $\left[\hbar_{i} \hbar_{j} \hbar_{k}\right]$ and $\mathbf{p}_{i}$ is a subset of $\mathbf{q}$ such that $\mathbf{q}^{\mathrm{T}}=\left[p_{i}^{\mathrm{T}} \mathbf{u}^{\mathrm{T}}\right]$, i.e. $\mathbf{u}^{\mathrm{T}}$ contains the remaining nonconstant generalized coordinates. A variety of the manifold is defined by

$\xi(\mathbf{u})=\xi\left(\mathbf{p}_{i}, \mathbf{u}\right)$ 
2. When certain parameters are at their limits there will be one zero row (one zero column) in $\mathbf{q}_{\lambda}$. The corresponding diagonal elements in matrix $\xi_{\mathbf{q}}$ will be equal to zero. Therefore, if $q_{i}, q_{j}$, and $q_{k}$ are at their limits, (e.g. $q_{i}=q_{i}^{\min }$ ) the diagonal elements of $\mathbf{q}_{\lambda}$ will be zero. Thus, the row-rank deficiency of $\mathbf{H}_{\mathbf{q}^{*}}$ depends on

$$
\boldsymbol{\Lambda}_{\mathbf{q}}=\left[\begin{array}{ccccccc}
\xi_{q_{1}} & \ldots & \xi_{q_{i}} & \xi_{q_{j}} & \xi_{q_{k}} & \ldots & \xi_{q_{n}} \\
0 & \ldots & 1 & \ldots & \ldots & \ldots & 0 \\
0 & \ldots & \ldots & 1 & \ldots & \ldots & 0 \\
0 & \ldots & \ldots & \ldots & 1 & \ldots & 0
\end{array}\right]
$$

which is a $(7 \times(n+m))$ matrix, with $\left[q_{i}, q_{j}, q_{k}\right]=\left[q_{i}^{\text {limit }}, q_{j}^{\text {limit }}, q_{k}^{\text {limit }}\right]$. The solution to this submatrix's row-rank deficiency condition is equivalent to solving the rank-deficiency for

$\left[\boldsymbol{\xi}_{\mathbf{q}} \oplus\left[\boldsymbol{\xi}_{q_{i}}, \boldsymbol{\xi}_{q_{j}}, \boldsymbol{\xi}_{q k}\right]\right]$

with $q_{i}=q_{i}^{\text {limit }}, q_{j}=q_{j}^{\text {limit }}, q_{k}=q_{k}^{\text {limit }}$

where the notation $\oplus$ represents the exclusion of the right matrix from the left matrix. Define a new vector $\partial \mathbf{q}^{\lim }$ as a sub-vector of $\mathbf{q}$, composed of joint coordinates that have reached their limits, i.e. $\partial \mathbf{q}^{\mathrm{lim}} \equiv\left[q_{i}^{\text {limit }}, q_{j}^{\text {limit }}, q_{k}^{\text {limit }}, \ldots\right]$ and $1 \leq \operatorname{dim}\left(\partial \mathbf{q}^{\lim }\right) \leq(n+m-3)$. Since $\partial \mathbf{q}^{\lim }$ is a constant vector, the remaining variables in $\mathbf{q}$ are denoted by $\mathbf{w}^{\mathrm{T}}$ such that

$\mathbf{q}=\left[\mathbf{w}^{\mathrm{T}}, \quad\left(\partial \mathbf{q}^{\lim }\right)^{\mathrm{T}}\right]^{\mathrm{T}}$, and $\mathbf{w} \cap \partial \mathbf{q}^{\mathrm{lim}}=\phi$

If $\mathbf{H}_{\mathbf{w}}\left(\mathbf{w}, \partial \mathbf{q}^{\text {lim }}\right)$ is row-rank deficient, the sub-Jacobian $\mathbf{H}_{\mathbf{q}^{*}}$ will also be rank-deficient. Let the solution for the rank deficiency of $\mathbf{H}_{\mathbf{w}}$ be $\hat{\mathbf{s}}$, which is a constant sub-vector of $\mathbf{w}$, and $\mathbf{w}=\left[\mathbf{u}^{\mathrm{T}}, \hat{\mathbf{s}}^{\mathrm{T}}\right]^{\mathrm{T}}$. The constant singular set is defined as $\mathbf{p}_{i}=\left[\hat{\mathbf{s}} \cup \partial \mathbf{q}^{\lim }\right]$ and the variety is again

$\boldsymbol{\xi}(\mathbf{u})=\boldsymbol{\xi}\left(\mathbf{p}_{i}, \mathbf{u}\right)$

For the case when $\operatorname{dim}\left(\partial \mathbf{q}^{\lim }\right) \leq(n+m-2)$, a combination of parameters at their limits is taken.

\section{References}

[1] Hoffman C. Geometric and solid modeling: an introduction, San Mateo, CA: Morgan Kaufmann, 1989.
[2] Abdel-Malek K, Yeh HJ, Othman S. Swept volumes: void and boundary identification. Computer Aided Design 1999;30(13): 1009-1018.

[3] Ahn JC, Kim MS, Lim SB. Approximate general sweep boundary of 2D curved object. CVGIP: Computer Vision Graphics and Image Processing 1997;55:98-128.

[4] Akman V, Arslan A. Sweeping with all graphical ingredients in a topological picturebook. Computers and Graphics 1992;16:273281.

[5] Elber G. Global error bounds and amelioration of sweep surfaces. Computer Aided Design 1997;29:441-447.

[6] Kieffer J, Litvin FL. Swept volume determination and interference detection for moving 3-D solids. Transactions of the ASME, Journal of Mechanical Design 1991;113:456-463.

[7] Kim MS, Moon SR. Rotational sweep volumes of objects bounded by algebraic curves. Proceedings of the IEEE International Conference on Robotics and Automation, 1990, p. 311-16.

[8] Leavitt J, Messcher W. Automatic Generation of 3D-envelopes. Proceedings of the NASA Symposium on Computer-Aided geometry Modeling, 1984, p. 145-49.

[9] Ling ZK, Chase T. Generating the swept area of a body undergoing planar motion. ASME Journal of Mechanical Design 1996;118:221233.

[10] Martin RR, Stephenson PC. Sweeping of three-dimensional Objects. Computer Aided Design 1990;22(4):223-234.

[11] Parida L, Mudur SP. Computational methods for evaluating swept object boundaries. Visual Computer 1994;10(5):266-276.

[12] Sambandan K, Wang KK. Five-axis swept volume for NC simulation and verification. ASME DE 1989;19:143-150.

[13] Schroeder WJ, Lorensen WE, Linthicum S. Implicit modeling of swept surfaces and volumes. Proceedings of the IEEE Visualization Conference. Los Alamitos, CA, 1994: 40-45.

[14] Sourin A, Pasko A. A function represenatation for sweeping by a moving solid. IEEE Transactions on visualization and Computer Graphics 1996;2:11-18.

[15] Wang WP, Wang KK. Geometric modeling for swept volume of moving solids. IEEE Computer Graphics and Applications 1986;6(12):8-17.

[16] Weld JD, Leu MC. Geometric representation of swept volumes with application to polyhedral objects. The International Journal of Robotics Research 1991;9(5):105-117.

[17] Abdel-Malek K, Yeh HJ. Geometric representation of the swept volume using Jacobian rank-deficiency conditions. Computer Aided Design 1997;29(6):457-468.

[18] Ganter MA, Storti DW, Ensz MT. On algebraic methods for implicit swept solids with finite extent. ASME Advances in Design Autoamtion, DE 1993;65(2):389-396.

[19] Blackmore D, Leu MC, Frank S. Analysis and modeling of deformed swept volumes. Computer Aided Design 1994;25(4):315-326.

[20] Blackmore D, Leu MC, Wang LP. Sweep-envelope differential equation algorithm and its application to NC machining verification. Computer Aided Design 1997;29(9):629-637.

[21] Blackmore D, Leu MC, Wang LP, Jiang H. Swept volumes: a retrospective and prospective view. Neural parallel and Scientific Computations 1997;5:81-102.

[22] Denavit J, Hartenberg RS. A kinematic notation for lower-pair mechanisms based on matrices. Transactions of ASME 1955;77: 215-221 Journal of Applied Mechanics, vol. 22..

[23] Paul RP. Robot manipulators: mathematics programming and control, Cambridge, Mass and London: MIT Press, 1981.

[24] Fu KS, Gonzalez RC, Lee CSG. Robotics: control, sensing, vision and intelligence. McGraw Hill, 1987. 


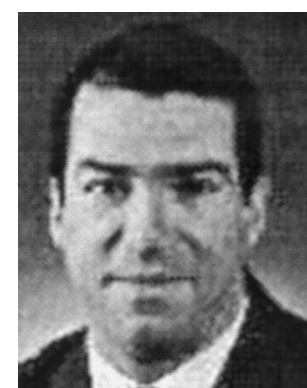

Karim Abdel-Malek is an Assistant Professor of Mechanical engineering at the University of Iowa. He received his $\mathrm{BS}$ degree in ME from the University of Jordan, his MS and PhD from the University of Pennsylvania, both in mechanical engineering. He was a Fulbright scholar for two years at the University of Pennsylvania. Dr Abdel-Malek consulted for the US manufacturing industry for a number of years before joining the faculty at Iowa. He served as a two-year appointment at the American University of Beirut in Beirut, Lebanon, on leave from Iowa. His research interests include mathematical formulations for CAD/CAM systems, robotics, dynamics, and machine design.

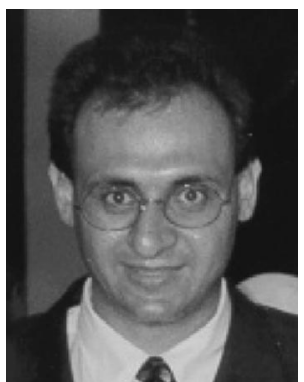

Saib Othman is a research engineer at the National Advanced Driving Simulator at the University of Iowa. He received his MS and $\mathrm{PhD}$ degrees in Mathematics from the University of Iowa and an MS degree in Mechanical Engineering. He is currently working on developing new terrain-vehicle mathematical models for simulating operator-in-the loop scenarios funded by the Department of Defense. His research interests are in computational mathematics, solid modeling, optimization, $\mathrm{CAD} / \mathrm{CAM}$, and computational dynamics. 